\title{
Quenching of highly vibrationally excited pyrimidine by collisions with $\mathrm{CO} 4$
}

Jeremy A. Johnson

jeremy.andrew.johnson@gmail.com

Andrew M. Duffin

Brian J. Hom

Karl E. Jackson

Eric T. Sevy

Follow this and additional works at: https://scholarsarchive.byu.edu/facpub

Part of the Biochemistry Commons, and the Chemistry Commons

\section{Original Publication Citation}

Johnson, Jeremy A., Andrew M. Duffin, Brian J. Hom, Karl E. Jackson, and Eric T.

Sevy."Quenching of highly vibrationally excited pyrimidine by collisions with $\mathrm{CO}[\mathrm{sub} 2]$." The Journal of Chemical Physics 128 (28).

\section{BYU ScholarsArchive Citation}

Johnson, Jeremy A.; Duffin, Andrew M.; Hom, Brian J.; Jackson, Karl E.; and Sevy, Eric T., "Quenching of highly vibrationally excited pyrimidine by collisions with CO4" (2008). Faculty Publications. 203.

https://scholarsarchive.byu.edu/facpub/203

This Peer-Reviewed Article is brought to you for free and open access by BYU ScholarsArchive. It has been accepted for inclusion in Faculty Publications by an authorized administrator of BYU ScholarsArchive. For more information, please contact ellen_amatangelo@byu.edu. 


\title{
Quenching of highly vibrationally excited pyrimidine by collisions with $\mathrm{CO}_{2}$
}

\author{
Jeremy A. Johnson, ${ }^{\text {a) }}$ Andrew M. Duffin, ${ }^{\text {b) }}$ Brian J. Hom, ${ }^{\text {c) }}$ Karl E. Jackson, ${ }^{\text {d) }}$ and \\ Eric T. Sevy \\ Department of Chemistry and Biochemistry, Brigham Young University, Provo, Utah 84602, USA
}

(Received 6 August 2007; accepted 26 November 2007; published online 6 February 2008)

Relaxation of highly vibrationally excited pyrimidine $\left(\mathrm{C}_{4} \mathrm{~N}_{2} \mathrm{H}_{4}\right)$ by collisions with carbon dioxide has been investigated using diode laser transient absorption spectroscopy. Vibrationally hot pyrimidine $\left(E^{\prime}=40635 \mathrm{~cm}^{-1}\right)$ was prepared by 248 -nm excimer laser excitation, followed by rapid radiationless relaxation to the ground electronic state. The nascent rotational population distribution $(J=58-80)$ of the $00^{0} 0$ ground state of $\mathrm{CO}_{2}$ resulting from collisions with hot pyrimidine was probed at short times following the excimer laser pulse. Doppler spectroscopy was used to measure the $\mathrm{CO}_{2}$ recoil velocity distribution for $J=58-80$ of the $00^{\circ} 0$ state. Rate constants and probabilities for collisions populating these $\mathrm{CO}_{2}$ rotational states were determined. The measured energy transfer probabilities, indexed by final bath state, were resorted as a function of $\Delta E$ to create the energy transfer distribution function, $P\left(E, E^{\prime}\right)$, from $E^{\prime}-E \sim 1300-7000 \mathrm{~cm}^{-1} . P\left(E, E^{\prime}\right)$ is fitted to a single exponential and a biexponential function to determine the average energy transferred in a single collision between pyrimidine and $\mathrm{CO}_{2}$ and parameters that can be compared to previously studied systems using this technique, pyrazine/ $\mathrm{CO}_{2}, \mathrm{C}_{6} \mathrm{~F}_{6} / \mathrm{CO}_{2}$, and methylpyrazine/ $\mathrm{CO}_{2} . P\left(E, E^{\prime}\right)$ parameters for these four systems are also compared to various molecular properties of the donor molecules. Finally, $P\left(E, E^{\prime}\right)$ is analyzed in the context of two models, one which suggests that the shape of $P\left(E, E^{\prime}\right)$ is primarily determined by the low-frequency out-of-plane donor vibrational modes and one which suggests that the shape of $P\left(E, E^{\prime}\right)$ can be determined by how the donor molecule final density of states changes with $\Delta E$. () 2008 American Institute of Physics.

[DOI: $10.1063 / 1.2825599$ ]

\section{INTRODUCTION}

Collisional energy transfer is the rate-limiting step in unimolecular reactions in low pressure or falloff regimes. ${ }^{1,2}$ Collisions between highly vibrationally excited molecules (with internal energies above or near threshold for unimolecular reaction) and thermal bath molecules play an important role in determining the fate of molecules poised to undergo decomposition or rearrangement. Competition between reaction and collisional energy transfer determines the overall kinetics and dynamics of chemical reactions. As such, studies of vibrational energy transfer from highly vibrationally excited molecules, possessing chemically significant amounts of energy, to cool bath molecules have received a great deal of attention. ${ }^{3}$ Of particular interest in understanding the relationship between unimolecular reaction and energy transfer is the energy transfer probability distribution function, $P\left(E, E^{\prime}\right)$, which gives the probability that a molecule initially at energy $E^{\prime}$ will, after a collision with a bath molecule, have an energy $E . P\left(E, E^{\prime}\right)$ is a key energy transfer

\footnotetext{
${ }^{a)}$ Present address: Department of Chemistry, Massachusetts Institute of Technology, Cambridge, MA 02139, USA.

${ }^{b)}$ Present address: Department of Chemistry, University of California, Berkeley, CA 94720, USA.

${ }^{c}$ Present address: McCampbell Analytical, Inc., 1534 Willow Pass Road, Pittsburg, CA 94565, USA.

${ }^{d)}$ Present address: Department of Chemistry, University of Washington, Seattle, WA 98195, USA.

e) Author to whom correspondence should be addressed. Electronic mail: esevy@byu.edu.
}

quantity because it describes the evolution of energy distributions during collisional activation and deactivation processes, as well as the various energy transfer moments. ${ }^{2}$

In the past ten years, three experimental techniques have been used to measure $P\left(E, E^{\prime}\right)$ for large molecules with chemically significant amounts of energy, including kinetically controlled selective ionization, ${ }^{4,5}$ a crossed molecular beam apparatus in conjunction with time-sliced velocity map imagining, ${ }^{6}$ and inversion of state resolved energy transfer data obtained using a high-resolution infrared diode laser probe technique..$^{7-9}$ In contrast to most experimental methods used to study energy transfer, the high-resolution IR diode laser probe technique developed by Flynn and co-workers ${ }^{7-12}$ measures the energy gained by bath molecules in a collision, rather than energy lost from donor molecules. Due to the high-frequency resolution of the diode lasers $\left(0.0003 \mathrm{~cm}^{-1}\right)$, it is possible to determine not only the average amount of energy transferred in a single donor/bath encounter, but also the amount of vibrational, rotational, and translational energies transferred in a collision. The energy transfer rate constant and probability can also be measured directly.

Of equal importance is the ability to directly invert the energy transfer probability, indexed by bath quantum state, into the energy transfer distribution function, $P\left(E, E^{\prime}\right)$, from $\sim 1300-7000 \mathrm{~cm}^{-1}{ }^{7-9} P\left(E, E^{\prime}\right)$ calculated for the pyrazine, ${ }^{7}$ $\mathrm{C}_{6} \mathrm{~F}_{6}{ }^{7,8}$ and methylpyrazine ${ }^{9}$ systems suggest that lowfrequency, out-of-plane donor molecule vibrational modes are responsible for the fraction of "supercollisions" (collision 
events in which large amounts of energy, $\sim 10 \mathrm{kT}$ or greater, are transferred in a single event), while proximity of the donor molecule to dissociation determines the magnitude of the strong collision. Using the diode-probe technique to study relaxation of highly vibrationally excited donors by $\mathrm{CO}_{2},{ }^{13-15}$ and a similar F-center laser probe technique to study relaxation by $\mathrm{H}_{2} \mathrm{O},{ }^{16}$ Mullin and co-workers have suggested that the change in donor density of states as a function of energy transferred mirrors the probability distribution function.

Much work has also gone into developing the theory to explain energy transfer processes. This effort includes classical trajectory studies, ${ }^{17-22}$ and quantum-scattering studies. ${ }^{21}$ Of particular importance to the work reported here are trajectory studies of Lendvay and co-worker ${ }^{17,20}$ and the work of Clary et al. ${ }^{21}$ They conclude that low-frequency donor vibrational modes, with out-of-plane-type motions, are more efficient at transferring large amounts of energy in collisions. Additional results from trajectory calculations by Lendvay et $a l{ }^{18}$ indicate that energy transfer efficiency as a function of donor molecule internal energy increases linearly at low internal energies; however, at high internal energies, approaching the threshold for dissociation, the dependence of energy transfer efficiency on internal energy becomes stronger than quadratic. This suggests that molecules ready to explode are more efficient at transferring energy than those farther from dissociation, even with the same internal energy.

Here we present a study of the collisional deactivation of vibrationally excited pyrimidine $\left(\mathrm{C}_{4} \mathrm{~N}_{2} \mathrm{H}_{4}, \quad E_{\text {int }}\right.$ $=40635 \mathrm{~cm}^{-1}$ ) by a bath of carbon dioxide using infrared transient absorption spectroscopy. Vibrationally excited pyrimidine is created by 248-nm UV absorption followed by radiationless relaxation to the ground electronic state, which is rapid ${ }^{23}$ compared to the mean donor/bath collision time. Absolute rate constants for the excitation of $\mathrm{CO}_{2}$ into high $J$ levels $(J=58-80)$ of the ground vibrational state are reported, along with the Doppler linewidths of these states. The energy transfer probabilities as a function of final $\mathrm{CO}_{2}$ quantum state are resorted as a function of energy to extract $P\left(E, E^{\prime}\right)$ from $\Delta E \sim 1300-7000 \mathrm{~cm}^{-1}$. This function is then fitted to both single exponential and biexponential functional forms to extract a value for the average energy transferred in a single collision of highly excited pyrimidine and $\mathrm{CO}_{2}$, $\langle\Delta E\rangle$, and the parameters that describe $P\left(E, E^{\prime}\right)$. The pyrimidine $/ \mathrm{CO}_{2} P\left(E, E^{\prime}\right)$ is then compared to previously studied donor $/ \mathrm{CO}_{2}$ systems at this internal energy to gain insight into factors that govern the shape of this function. In particular, comparison is made between molecules at or near threshold for unimolecular dissociation and those well below threshold.

\section{EXPERIMENTAL}

The UV pump, IR probe technique used to study the collisional relaxation of pyrimidine by collisions with $\mathrm{CO}_{2}$ has been described in detail elsewhere; ${ }^{11}$ therefore, only a brief outline of the method is presented here. A more complete description of the specifics of our experimental setup can be found in the Appendix. ${ }^{24}$ A 1:1 mixture of gas phase pyrimidine and $\mathrm{CO}_{2}$ flows through a $3.0 \mathrm{~m}$ Pyrex collision cell at a total pressure of 20 mTorr. A 248-nm KrF excimer laser is used to excite the $S_{2} \leftarrow S_{0}$ transition of pyrimidine. Excitation to the excited singlet state results in rapid intersystem crossing to the excited vibrational levels of the triplet state, which are in turn strongly coupled to the highly vibrationally excited levels of the ground electronic state. The lifetime of triplet excited pyrimidine following 266-nm excitation has been measured to be $\sim 30 \mathrm{~ns}{ }^{23}$ Following 248-nm excitation, we expect the triplet state lifetime to be shorter than $30 \mathrm{~ns}$, which is much shorter than the mean collision time of pyrimidine $/ \mathrm{CO}_{2}$ studied here. Energy gain into individual rotational states of the $\mathrm{CO}_{2}\left(00^{0} 0\right)$ vibrational state resulting from collisions with hot pyrimidine in $S_{0}{ }^{*}$ is monitored by probing the transient absorption of IR light $(\lambda$ $=4.3 \mu \mathrm{m})$ that is collinearly propagated with the UV beam through the collision cell. The highly resolved $\left(0.0003 \mathrm{~cm}^{-1}\right)$ lead salt IR diode laser is used to probe the scattered $\mathrm{CO}_{2}$ molecules via the antisymmetric stretch transition of $\mathrm{CO}_{2}$. To ensure that only a single diode laser mode is detected, the infrared light is passed through a single grating monochromator before being focused onto a liquid nitrogen cooled $\mathrm{InSb}$ detector. The detector and preamplifier combination has a rise time of approximately $400 \mathrm{~ns}$, shorter than 1/4 the gas kinetic collision time $(1 \mu \mathrm{s})$. The signal from the InSb detector is digitized and stored on a digital oscilloscope before being transferred to a computer for further analysis. Approximately $4 \%$ of the infrared light is split off and passed through a reference line. The reference beam passes through a second monochromator and is focused onto a high gain $\mathrm{InSb}$ detector. The output of the reference detector is used as input to a lock-in amplifier, which generates an error signal that is fed back to the diode laser control electronics allowing active stabilization of the laser frequency.

Determination of rovibrational state populations requires a measurement of both the transient absorption at the center of the line and the Doppler broadened lineshape; therefore, two types of measurements are collected for each rotational state: the transient fractional absorption, $\Delta I / I$, after $1 \mu \mathrm{s}$ $(1 / 4$ the gas kinetic collision time of $4 \mu \mathrm{s})$ at the center frequency of the transition, and a measure of the Dopplerbroadened full width at half maximum (FWHM). The center line measurement is obtained by locking the diode laser frequency to the appropriate $\mathrm{CO}_{2}$ line and averaging the absorption over approximately 100 excimer laser shots. A dual channel technique $^{10}$ is employed to account for short term fluctuations in the diode laser intensity, while a reference line scheme $^{25}$ is used with the center line measurements to correct for longer term drifts in the system. A short cell reference procedure is performed to determine the absolute scattering rate constant. In addition, to ensure that scattering results can be accurately compared to previously studied systems, two $3 \mathrm{~m}$ cells are used to obtain transient signals for pyrimidine $/ \mathrm{CO}_{2}$ scattering, followed closely in time by a probe of pyrazine $/ \mathrm{CO}_{2}$ scattering. The pyrazine $/ \mathrm{CO}_{2}$ scattering results from this long cell experiment are compared to previously published work ${ }^{10}$ and a scaling factor determined. 
This ensures that pyrimidine relaxation results can be meaningfully compared to results from previously studied systems. $^{8-10}$

Lineshape measurements are performed by locking the diode laser frequency to the peak of a scanning Fabry-Pérot etalon fringe (free spectral range $=289 \mathrm{MHz}$ ). Absorption by $\mathrm{CO}_{2}$ is then measured at a series of 30-40 frequencies distributed evenly over the lineshape, averaged over 100 excimer laser shots at each frequency.

Recently ${ }^{26}$ it was shown that pyrimidine undergoes dissociation following 248-nm excitation. To ensure that this photochemistry is not interfering with the energy transfer results, precautions were taken similar to those for pyrazine, ${ }^{27,28}$ which dissociates on the same time scale. In particular, a fast InSb detector and preamplifier with a $10 \mathrm{~ns}$ rise time was used in place of the $400 \mathrm{~ns}$ rise time signal detector to measure the transient absorption signal. The fast response of this scheme allowed us to obtain the singlecollision energy transfer transient absorption measurements with higher $\mathrm{CO}_{2}$ gas pressures (i.e., shorter mean collision times) than studied with the $400 \mathrm{~ns}$ rise time detectorpreamplifier combination. This shorter mean collision time allows us to probe the energy transfer process well before photodissociation can interfere with results. Both the transient absorption signal and the Doppler-broadened lineshape were measured using this fast detector-amplifier setup.

Pyrimidine (Aldrich, 99+\%), pyrazine (Aldrich, 99+\%), and research-grade $\mathrm{CO}_{2}$ (Intermountain Airgas Inc., 99.999\%) were used in these experiments. Pyrimidine and pyrazine were degassed using no less than three freeze $(77 \mathrm{~K}) /$ pump/thaw cycles before use, while $\mathrm{CO}_{2}$ was used without further purification.

\section{RESULTS AND DISCUSSION}

\section{A. Fast detection of $\mathrm{CO}_{2}\left(00^{\circ} 0,70, v\right)$}

Lin et $a .^{26}$ have recently studied the photodissociation dynamics of pyrimidine using multimass ion imaging techniques following 248-nm photodissociation. They observed several pyrimidine photodissociation channels at this wavelength. Although the nature of their experiment precludes measurements of the photodissociation quantum yields, they were able to determine the dissociation rate constant of pyrimidine at this energy. Their measured lifetime is on the microsecond time scale similar to pyrazine $e^{27,28}$ at this energy. They also studied the photolysis as a function of laser intensity in the range of $0.6-11 \mathrm{~mJ} / \mathrm{cm}^{2}$, which indicated that 248-nm photodissociation was the result of single photon absorption.

Because pyrimidine dissociates following 248-nm excitation, great care was needed in the energy transfer experiments to ensure that the observed excitation of $\mathrm{CO}_{2}$ is due to collisions with vibrationally hot pyrimidine, rather than excited pyrimidine photoproducts. This is particularly important in bath probe experiments, since bath molecules are blind to the identity of the donor molecules in energy transfer processes. Studies ${ }^{28}$ of pyrazine dissociation and its effect on energy transfer concluded that multiphoton UV absorption was responsible for the majority of photoproduct produced

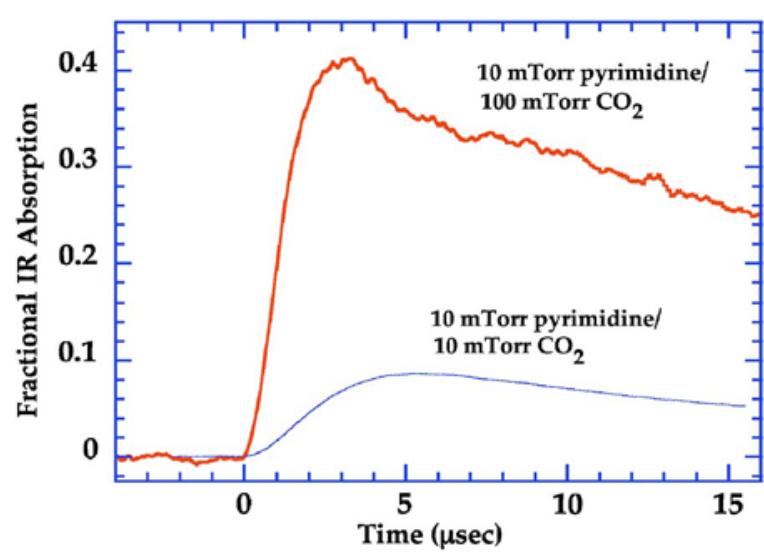

FIG. 1. (Color online) The fractional IR absorption of $\mathrm{CO}_{2}$ collisionally scattered into the $00^{\circ} 0, J=70$ state. The lower curve was obtained using a

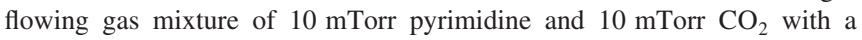
mean kinetic collision time of $4 \mu \mathrm{s}$. The upper curve was collected using a fast-rise-time detector/amplifier and a mixture of 10 mTorr pyrimidine and 100 mTorr $\mathrm{CO}_{2}$ with a mean collision time of $800 \mathrm{~ns}$. The absorption is linear in both cases for the early time regime, indicating that population of the high $\mathrm{CO}_{2} J$ states at early times is the result of collisions with vibrationally excited pyrimidine.

on a time scale and with sufficient energy to interfere with energy transfer results; therefore, power dependent studies were performed to ensure that pyrimidine UV absorption was in the linear region and photodissociation due to multiphoton absorption was eliminated. Pyrimidine UV absorption increases linearly with laser intensity from 4 to $29 \mathrm{~mJ} / \mathrm{cm}^{2}$; all energy transfer studies were performed at a laser intensity of $4-5 \mathrm{~mJ} / \mathrm{cm}^{2}$, resulting in a typical fraction of excited pyrimidine molecules of $2 \times 10^{-5}$.

Two additional experiments, designed to distinguish energy transfer involving hot pyrimidine from energy transfer as a result of hot photoproducts, were performed. These studies are modeled after previous pyrazine work $^{27}$ and are designed to take advantage of the difference in the time dependence of the vibrationally excited pyrimidine population and the time scale of photoproduct production. Because of the rapid time in which vibrationally excited pyrimidine is produced $(<30 \mathrm{~ns}),{ }^{23}$ the vibrationally excited pyrimidine population is maximized at early times. The excited pyrimidine population then decays, either by collisional relaxation or by photodissociation. In contrast, the UV photoproduct population, initially at zero, increases only at longer times. Using a detector with a fast response time, it is possible to study the energy transfer process before significant amounts of photoproduct are produced and determine the identity of the donor molecule in collisions that excited $\mathrm{CO}_{2}$ into high angular momentum states.

In the first experiment, transient absorption signals taken under conditions that significantly change the mean collision time between $\mathrm{CO}_{2}$ and pyrimidine are compared. Typical transient signals are shown in Fig. 1, where absorption on the $\mathrm{CO}_{2}\left(00^{0} 0, J=70\right) \rightarrow \mathrm{CO}_{2}\left(00^{0} 1, J=69\right)$ line at $2279.8391 \mathrm{~cm}^{-1}$ is plotted against time relative to the excimer laser pulse. The lower curve is the transient absorption average over 100 excimer laser shots fired into a flowing gas mixture of 10 mTorr pyrimidine and $10 \mathrm{mTorr} \mathrm{CO}_{2}$, collected using a $400 \mathrm{~ns}$ rise time detector, while the upper 
curve is the transient absorption of a 10 mTorr pyrimidine and 100 mTorr $\mathrm{CO}_{2}$ mixture, collected with a fast detector with a $10 \mathrm{~ns}$ rise time. The two transient signals differ in the pressure of $\mathrm{CO}_{2}$ and thus in the mean collision time involved in the energy transfer process. The behavior of both of these signals is linear at early times, in particular, at $1 / 4$ the gas kinetic collision time, the time at which IR absorption signals are used in the energy transfer calculations. A detailed analysis of the kinetics for transient absorption measurements performed for pyrazine/ $\mathrm{CO}_{2}$ (Ref. 27) shows that collisional excitation of the high $J$ states of $\mathrm{CO}_{2}$ by hot donor molecules at early times following UV excitation is linear in time; however, transient absorption measurements of $\mathrm{CO}_{2}$ produced via collisions with hot photoproducts scale as $t^{2}$. Furthermore, the energy transfer rate constants calculated from the two transients in Fig. 1 are the same within experimental error, supporting the conclusion that in both the fast and slow experiments the energy transfer process is a result of $\mathrm{CO}_{2}$ colliding with hot pyrimidine.

The second experiment is a measure of the Dopplerbroadened lineshape for the absorption transition $\mathrm{CO}_{2}\left(00^{0} 0,70\right) \rightarrow \mathrm{CO}_{2}\left(00^{0} 1,69\right)$. Figure 2(a) shows a transient absorption lineshape obtained from a measurement taken at $1 \mu$ s after excimer laser firing through a flowing mixture of 10 mTorr of pyrimidine and $10 \mathrm{mTorr}$ of $\mathrm{CO}_{2}$; Fig. 2(b) shows a similar lineshape obtained from a measurement $200 \mathrm{~ns}$ after excimer laser firing through a flowing mixture of $10 \mathrm{mTorr}$ of pyrimidine and $100 \mathrm{mTorr}$ of $\mathrm{CO}_{2}$. The linewidth (FWHM) obtained from both experiments is $\Delta \nu$ $\sim 0.010 \pm 0.001 \mathrm{~cm}^{-1}$, the same within experimental error. These extremely broadened absorption lineshapes indicate the presence of very large $V \rightarrow T$ energy transfers, regardless of the species responsible for the excitation. The basic idea of this experiment is to greatly reduce the amount of photoproduct in the cell while mildly increasing the amount of vibrationally hot pyrimidine. This is accomplished by simply probing $\mathrm{CO}_{2}$ at significantly shorter times (200 ns vs $1 \mu \mathrm{s}$ ). The signature of large energy transfer (a broad linewidth) is present at short times as well as long. Qualitatively this suggests that vibrationally hot pyrimidine is the likely donor molecule in these large energy transfer collisions, as they seem to persist in an environment where the amount of photoproduct is reduced.

All three experiments are consistent with energy transfer to $\mathrm{CO}_{2}$ resulting from collisions with vibrationally excited pyrimidine rather than hot photoproducts; thus, the energy transfer studies presented in this paper are the result of collisional deactivation of pyrimidine poised to undergo unimolecular decomposition. These studies can be compared to collisional deactivation of pyrazine,${ }^{10}$ also near its dissociation threshold at $248 \mathrm{~nm}$ and contrasted with collisional deactivation studies of methylpyrazine ${ }^{9}$ and $\mathrm{C}_{6} \mathrm{~F}_{6},{ }^{8}$ donors that are below threshold at this energy.

\section{B. Rotational and translational excitations of the carbon dioxide bath}

Transient $\mathrm{CO}_{2}$ populations in various $J$ states of the ground vibrational level, ranging from $J=58$ to 80 , were
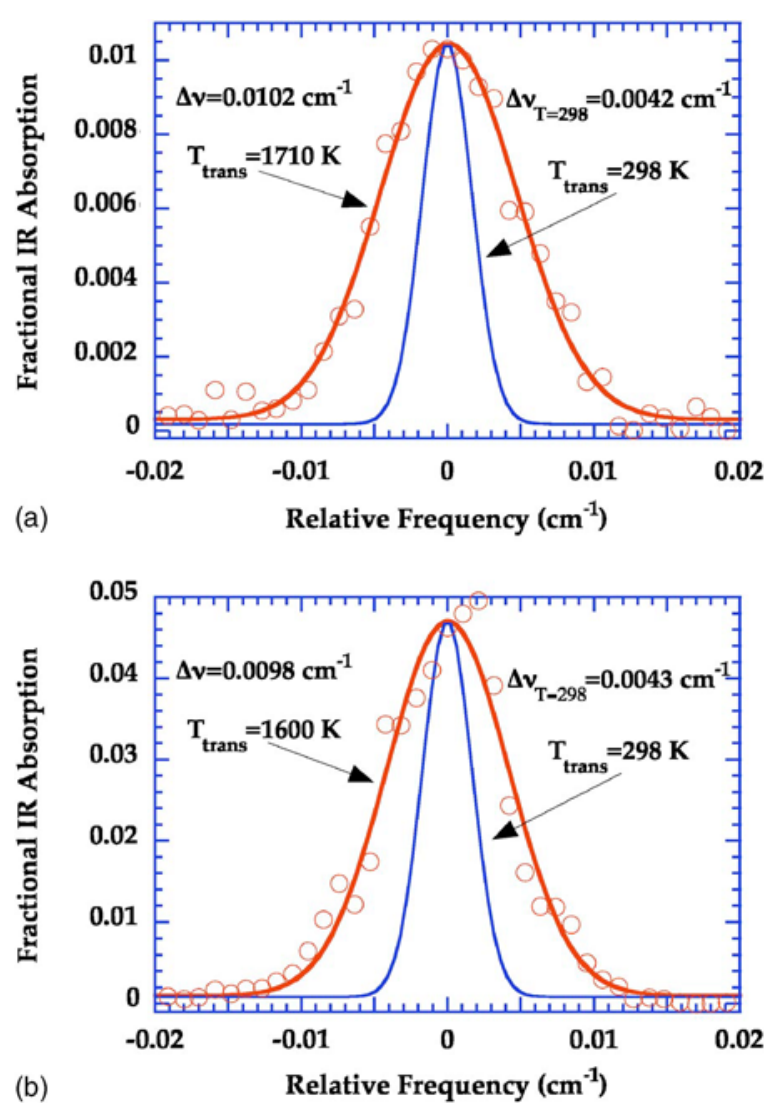

FIG. 2. (Color online) Nascent Doppler broadened lineshapes for the absorption transition $\mathrm{CO}_{2}\left(00^{0} 0 ; J=70\right) \rightarrow \mathrm{CO}_{2}\left(00^{0} 1 ; J=69\right)$ probing $\mathrm{CO}_{2}$ molecules excited by collisions with vibrationally excited pyrimidine. The lineshapes were obtained using a flowing mixture of $10 \mathrm{mTorr}$ pyrimidine and (a) $10 \mathrm{mTorr} \mathrm{CO}_{2}$ or (b) $100 \mathrm{mTorr} \mathrm{CO}_{2}$. The points represent the fractional IR absorption of $\mathrm{CO}_{2}$ collisionally scattered in the $00^{\circ} 0, J=70$ state measured (a) $1 \mu \mathrm{s}$ and (b) $200 \mathrm{~ns}$ following 248-nm excimer laser pumping of pyrimidine. Absorption measurements are averaged over approximately 100 excimer laser shots fired at $1 \mathrm{~Hz}$ at each frequency across the line. The solid lines are the best nonlinear least squares fit to a Gaussian function. The linewidth obtain from each lineshape is $0.010 \pm 0.001$. For comparison, the FWHM of a $\mathrm{CO}_{2}$ lineshape with $T_{\text {trans }}=300 \mathrm{~K}$ is approximately $0.0042 \mathrm{~cm}^{-1}$ and indicated in each figure by the narrower Gaussian function.

monitored via infrared absorption following 248-nm excitation of pyrimidine. Because the $\mathrm{CO}_{2}$ rotational $^{29}$ and translational ${ }^{30}$ energies change with each collision, the distributions probed at short times after the excimer laser pulse represent the nascent collision dynamics without significant relaxation. Typical transient signals are shown in Fig. 1. As discussed above, the early, linear rise in the signals result from excitation of $\mathrm{CO}_{2}$ to $J=70$ by single collisions with vibrationally hot pyrimidine, rather than collisions between $\mathrm{CO}_{2}$ and hot photoproducts produced by UV photodissociation of pyrimidine, ${ }^{26}$ which would have a $t^{2}$ dependence. ${ }^{27}$ The time dependence of the signal at longer times $(t>4 \mu \mathrm{s}$ for the 10 mTorr: 10 mTorr mixture) is dictated by the competition between the continued filling of this state by collisions with hot pyrimidine molecules, which are on average only slightly thermalized or pyrimidine UV photoproducts, and $\mathrm{CO}_{2}$ rotational relaxation out of this high angular momentum state. Strictly speaking, the absorption signal is a measure of the change in the population difference between the initial and final states of the probe transition. Earlier ex- 


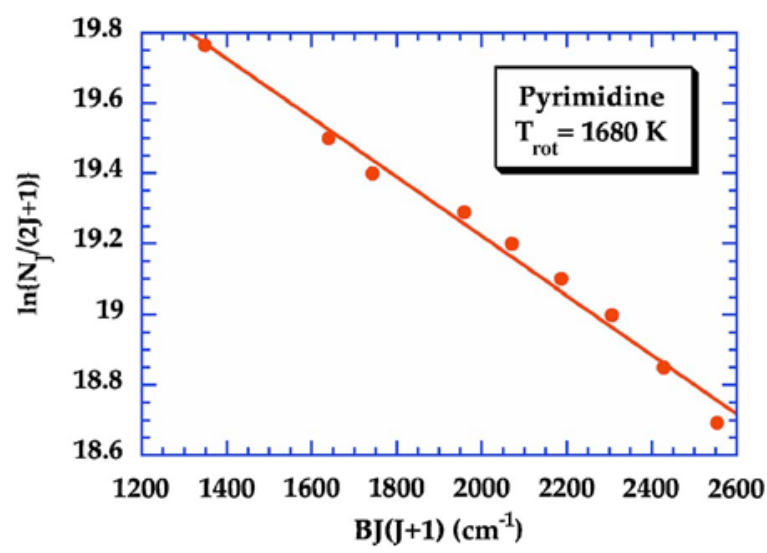

FIG. 3. (Color online) Boltzmann plot of the nascent rotational distributions in the ground vibrational level of $\mathrm{CO}_{2}$ following excitation by collisions with vibrationally hot pyrimidine. The solid line is the best linear least squares fit to the experimental points measured over the range $J=58-80$. The slope of the best-fit line is equal to $-1 / k T_{\text {rot }}$ and the number densities, $N(J)$, are in units of molecules $/ \mathrm{cm}^{3}$. The rotational temperature for $\mathrm{CO}_{2}$ molecules scattered into the high $J$ states is $1660 \pm 80 \mathrm{~K}$.

periments on the pyrazine/ $\mathrm{CO}_{2}$ and $\mathrm{C}_{6} \mathrm{~F}_{6} / \mathrm{CO}_{2}$ systems reveal that the number density of molecules scattering into the high $J$ tail of the $00^{0} 1$ state is essentially zero; ${ }^{31}$ thus, the measured absorptions simply reflect a transient increase in the population of molecules in the high $J$ tail of the ground vibrational level. Conversion of the measured absorptions to $\mathrm{CO}_{2}$ number densities is straightforward ${ }^{32}$ given the absorption linestrengths taken from the HITRAN spectroscopic database. $^{33}$

The rotational distributions over this range of final $J$ states are well described by a rotational temperature. A Boltzmann plot of the rotational distribution created by collisions of $\mathrm{CO}_{2}$ with hot pyrimidine is shown in Fig. 3. The indicated temperature only describes the high $J$ tail $(J=58-80)$ of the rotational distribution and may not be appropriate for molecules scattering into lower $J$ states. In fact, there is no reason to expect that a temperature will necessarily provide a suitable description of any part of the rotational distribution created by collisions with a vibrationally hot molecule; however, because a single temperature does characterize these high $J$ states of $\mathrm{CO}_{2}\left(00^{0} 0\right)$, temperature is a convenient measure of the amount of rotational excitation. The measured rotational temperature of $1660 \pm 80 \mathrm{~K}$ indicates that $\mathrm{CO}_{2}$ molecules are scattered into the high $J$ states in collisions with pyrimidine and that a large amount of energy is transferred in a single collision. This rotational temperature is larger than has been reported elsewhere for energy transfer from other highly vibrationally excited systems to $\mathrm{CO}_{2}$ (methylpyrazine ${ }^{9}-725 \mathrm{~K}, \quad \mathrm{C}_{6} \mathrm{~F}_{6} \quad$ (Ref. 8)-795 K, and pyrazine $\left.^{10}-1300 \mathrm{~K}\right)$.

Although the significantly larger $T_{\text {rot }}$ for $\mathrm{CO}_{2}$ following collisions with pyrimidine than any of the donor molecules is initially surprising, it should be noted that there is a smaller difference between the rotational temperature describing collisions with pyrimidine and pyrazine than between pyrazine and $\mathrm{C}_{6} \mathrm{~F}_{6}$. The large gap between the rotational temperatures describing the $\mathrm{C}_{6} \mathrm{~F}_{6}$ excited $\mathrm{CO}_{2}$ population and the pyrazine excited $\mathrm{CO}_{2}$ population may initially lead one to suspect that the photochemistry of pyrazine and pyrimidine is responsible for the larger $\mathrm{CO}_{2}$ rotational temperatures. However, a comparison of $\mathrm{CO}_{2}$ rotational temperature following collisions with several donor molecules shows that there is a nearly one-to-one correlation between $T_{\text {rot }}$ and the shape of the large $\Delta E$ tail of $P\left(E, E^{\prime}\right) .{ }^{34}$ As will be discussed in detail below, the shape of the large $\Delta E$ tail of $P\left(E, E^{\prime}\right)$ is related to how the final density of states of the donor molecule changes with energy. This suggests that the bath rotational temperature is related to how the donor state density changes with energy.

The projections of the distributions of $\mathrm{CO}_{2}$ lab frame, recoil velocities onto the probe beam axis were determined from measurements of the Doppler-broadened lineshape of the probe transitions. As seen in Fig. 2, a Gaussian function accurately fits the measured lineshapes, indicating that a single translational temperature is suitable to describe the $\mathrm{CO}_{2}$ translational excitation. Figure 2(a) shows the transient absorption lineshape obtained from a measurement taken at $1 \mu \mathrm{s}$ after excimer laser excitation of a mixture of $10 \mathrm{mTorr}$ of pyrimidine and 10 mTorr of $\mathrm{CO}_{2}$. The linewidth (FWHM) obtained from probing $\mathrm{CO}_{2}$ scattered into $J=70$ is $\Delta \nu$ $=0.0102 \pm 0.0006 \mathrm{~cm}^{-1}$, corresponding to a lab frame temperature of $1710 \mathrm{~K}$. For comparison purposes, the linewidth of a transition probing $\mathrm{CO}_{2}$ molecules with a room temperature velocity distribution, $\Delta \nu_{T=298}=0.0042 \mathrm{~cm}^{-1}$, is also shown. Figure 2(b) was obtained using the fast-rise-time detector at $200 \mathrm{~ns}$ after excimer laser excitation of mixture of 10 mTorr of pyrimidine and 100 mTorr of $\mathrm{CO}_{2}$. In this case, the linewidth obtained from probing $\mathrm{CO}_{2}$ scattered into $\mathrm{J}$ $=70$ is $\Delta \nu=0.0098 \pm 0.0007 \mathrm{~cm}^{-1}$, corresponding to a lab frame temperature of $1600 \mathrm{~K}$. Given the error bars on this measurement, these results likely correspond to the same linewidth and translational temperature, as well as the same amount of translational energy transfer, as discussed above. Pyrazine $/ \mathrm{CO}_{2}$ experiments ${ }^{10}$ indicate that postcollision velocity distributions are isotropic; therefore, the Dopplerbroadened lineshapes resulting from collisional energy transfer describe the three dimensional speed distribution of the $\mathrm{CO}_{2}$ molecules following collisions with highly vibrationally excited donors. Since fast detection studies indicate that excitation of $\mathrm{CO}_{2}$ in these studies results from collisions with vibrationally hot pyrimidine rather than hot photoproducts, it is reasonable to assume that the measured translational temperatures represent the three dimensional $\mathrm{CO}_{2}$ speed distribution, as in other energy transfer studies. ${ }^{8,9}$

Full-width, half-maximum Doppler linewidths for the absorption transitions probing scattering into $J$ states between 58 and 80 are given in Table I. Note that these linewidths are for the $10 / 10 \mathrm{mT}$ Torr pyrimidine $/ \mathrm{CO}_{2}$ mixture measured at $t=1 \mu \mathrm{s}$ after the excimer laser pulse, onequarter the mean gas kinetic collision time. The translational temperature of $\mathrm{CO}_{2}$ molecules recoiling from excited pyrimidine, also contained in Table I, is determined from the fitted lineshape using the relation

$$
T_{f}^{\mathrm{CO}_{2}}=\frac{m c^{2}\left(\Delta \nu_{\mathrm{obs}}\right)^{2}}{8 R \ln 2\left(\nu_{0}\right)^{2}},
$$

where $m$ is the mass of $\mathrm{CO}_{2}, c$ is the speed of light, $\Delta \nu_{\mathrm{obs}}$ is the fitted lineshape (full width at half maximum), $R$ is the gas 
TABLE I. Full width at half maximum Doppler linewidths of absorption transitions, translational temperatures, and center of mass translational temperatures for postcollision $\mathrm{CO}_{2}\left(00^{0} 0, j\right)$ levels for the process pyrimidine ${ }^{E^{\prime}}+\mathrm{CO}_{2}\left(00^{0} 0\right) \rightarrow$ pyrimidine ${ }^{E^{\prime}}+\mathrm{CO}_{2}\left(00^{0}, J\right)$ (the internal energy of pyrimidine following 248-nm excitation is $\left.E^{\prime}=40635 \mathrm{~cm}^{-1}\right)$.

\begin{tabular}{cccc}
\hline \hline Final $\mathrm{CO}_{2}$ rotational level, $J$ & $\Delta \nu_{\mathrm{obs}}\left(\mathrm{cm}^{-1}\right)^{\mathrm{a}}$ & $T_{f}^{\mathrm{CO}_{2}}(\mathrm{~K})^{\mathrm{b}}$ & $T_{f}^{\mathrm{c} . \mathrm{m} .}(\mathrm{K})^{\mathrm{c}}$ \\
\hline 58 & $0.0086 \pm 0.0008$ & $1200 \pm 230$ & $1690 \pm 360$ \\
64 & $0.0085 \pm 0.0007$ & $1180 \pm 200$ & $1660 \pm 310$ \\
66 & $0.0095 \pm 0.0007$ & $1500 \pm 230$ & $2160 \pm 350$ \\
70 & $0.0102 \pm 0.0006$ & $1710 \pm 210$ & $2490 \pm 320$ \\
72 & $0.0106 \pm 0.0006$ & $1840 \pm 220$ & $2690 \pm 330$ \\
74 & $0.0117 \pm 0.0006$ & $2260 \pm 240$ & $3340 \pm 370$ \\
76 & $0.0112 \pm 0.0006$ & $2100 \pm 230$ & $3090 \pm 360$ \\
80 & $0.0119 \pm 0.0005$ & $2350 \pm 200$ & $3480 \pm 310$ \\
$4600 \pm 360$ & $3080 \pm 230$ & 4600 \\
\hline
\end{tabular}

$\overline{\mathrm{a}}$ The measured full width at half maximum of the transient Doppler linewidths for the transitions $\mathrm{CO}_{2}\left(00^{0}, J\right) \rightarrow \mathrm{CO}_{2}\left(00^{0}, 1, J-1\right)$, determined at $1 \mu \mathrm{s}$ after pyrimidine excitation in a 1:1 sample of pyrimidine: $\mathrm{CO}_{2}$ at a total pressure of $20 \mathrm{mTorr}$. The thermal linewidth for $\mathrm{CO}_{2}$ at $T=298 \mathrm{~K}$ is $\Delta \nu_{0}$ $=0.0042 \mathrm{~cm}^{-1}$.

${ }^{{ }^{\mathrm{b}}}$ The final translational temperatures, $T_{f}^{\mathrm{CO}_{2}}$, are obtained from fitting the experimentally determined Doppler lineshapes with a Gaussian function, and are related to the linewidth (full width at half maximum), $\Delta \nu_{\mathrm{obs}}$, through the expression $T_{f}^{\mathrm{CO}_{2}}=\left[m c^{2}\left(\Delta \nu_{\mathrm{obs}}\right)^{2}\right] /\left[8 R \ln 2\left(\nu_{0}\right)^{2}\right]$, where $m$ is the mass of $\mathrm{CO}_{2}, c$ is the speed of light, $R$ is the gas constant, and $\nu_{0}$ is the wave number at the center of the absorption line.

${ }^{\mathrm{c}}$ The final center of mass translational temperatures, $T_{f}^{\mathrm{c} . \mathrm{m}}$. are obtained from the transient translational temperatures from the expression $T_{f}^{\mathrm{c} . \mathrm{m}}=T_{f}^{\mathrm{CO}}{ }_{2}+\left(T_{f}^{\mathrm{CO}_{2}}\right.$ $-T)\left(m_{\mathrm{CO}_{2}} / m_{\text {pyrimidine }}\right)$, where $T_{f}^{\mathrm{CO}_{2}}$ is the temperature describing the lab frame velocity, $T$ is the ambient cell temperature, and $m$ is the mass of $\mathrm{CO}_{2}$ and pyrimidine, respectively.

constant, and $\nu_{0}$ is the frequency at the center of the absorption line. As has been seen previously, $\mathrm{CO}_{2}$ molecules rotationally excited into high $J$ states by collisions with hot donor molecules also possess a large amount of translational energy. Assuming the final velocity distributions are isotropic, ${ }^{10}$ the temperature that describes the distribution of pyrimidine $/ \mathrm{CO}_{2}$ relative (center of mass) velocities, more relevant for determining the actual energy transfer in these collisions, can be determined from the measured, lab frame translational temperatures according to

$$
T_{f}^{\mathrm{c} . \mathrm{m} .}=T_{f}^{\mathrm{CO}_{2}}+\left(T_{f}^{\mathrm{CO}_{2}}-T\right)\left(\frac{m_{\mathrm{CO}_{2}}}{m_{\text {pyrimidine }}}\right) \text {, }
$$

where $T_{f}^{\text {c.m. }}$ is the pyrimidine/ $\mathrm{CO}_{2}$ center of mass translational temperature, $T_{f}^{\mathrm{CO}_{2}}$ is the temperature describing the $\mathrm{CO}_{2}$ lab frame velocity distribution, $T$ is the cell temperature $(298 \mathrm{~K})$, and the $m_{i}$ are the respective masses of the collision partners. The postcollision values of $T_{f}^{\mathrm{c} . \mathrm{m}}$. for $\mathrm{CO}_{2}\left(00^{0} 0\right) \mathrm{J}$ $=58$ to $\mathrm{J}=80$ are also listed in Table $\mathrm{I}$.

Several notable features of the linewidth data in Table I should be highlighted. First, the broadened linewidths indicate that collisions scattering molecules into high angular momentum states produce broad (high temperature) velocity distributions, indicating that large translational energy transfers accompany these collisions. Second, the linewidths vary nearly linearly with the final rotational angular momentum, as illustrated graphically in Fig. 4. This linear relationship was observed in the pyrazine/ $\mathrm{CO}_{2}$ (Refs. 10 and 12) and methylpyrazine/ $\mathrm{CO}_{2}$ (Ref. 9) systems but not in the $\mathrm{C}_{6} \mathrm{~F}_{6} / \mathrm{CO}_{2}$ system. $^{8}$ The linear variation of linewidth with $J$ signifies that these collisions, which impart large amounts of rotational and translational energies to $\mathrm{CO}_{2}$, sample the steep repulsive wall of the pyrimidine $/ \mathrm{CO}_{2}$ intermolecular potential energy surface and do so with an essentially constant impact parameter. ${ }^{9,12}$

\section{Final state resolved energy transfer rate constants and probabilities}

At short times, those much less than the mean gas kinetic collision time, the population of $\mathrm{CO}_{2}$ molecules in a particular $J$ state of the ground vibrational level can be written as follows:

$$
\left[\mathrm{CO}_{2}\left(00^{0} 0, J, V\right)\right]=k_{2}^{J}\left[\mathrm{CO}_{2}\right]_{0}\left[\text { pyrimidine }^{E^{\prime}}\right]_{0} t,
$$

where $k_{2}^{J}$ is the state specific rate constant for excitation of $\mathrm{CO}_{2}$ from a thermal distribution into state $J,\left[\mathrm{CO}_{2}\right]_{0}$ is the bulk carbon dioxide number density, and [pyrimidine $\left.E^{E^{\prime}}\right]_{0}$ is the number density of pyrimidine molecules excited by the excimer laser pulse to an energy $E^{\prime}$. The state specific $\mathrm{CO}_{2}$ number density is obtained from the infrared absorption measurements and the excited pyrimidine number density is

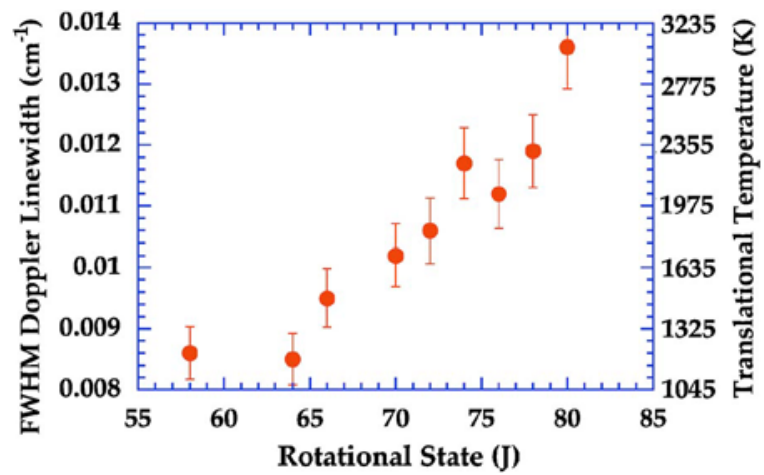

FIG. 4. (Color online) The FWHM Doppler linewidth of absorption transitions probing high rotational states of $\mathrm{CO}_{2}$ following excitation by collisions with vibrationally hot pyrimidine $\left(E^{\prime}=40635 \mathrm{~cm}^{-1}\right)$ is plotted against the final rotational state $J$. The lab frame translational temperatures, which are determined from the measured linewidths, are given on the right $y$-axis. The linewidths are measured $1 \mu$ s following 248-nm excimer laser excitation of the pyrimidine, ensuring that the measured velocity distributions are nascent $\left(\tau_{\text {coll }} \approx 4 \mu \mathrm{s}\right)$. The FWHM appropriate for a $298 \mathrm{~K}$ velocity distribution is $0.0042 \mathrm{~cm}^{-1}$. 
TABLE II. Rate constants (given in units of $\mathrm{cm}^{3}$ molecule $\left.{ }^{-1} \mathrm{~s}^{1 \prime}\right)\left(k_{2}^{J}\right)$ and probabilities $\left(\operatorname{Prob}_{\mathrm{LJ}}=k_{2}^{J} / k_{\mathrm{LJ}}\right)$ for the energy transfer process pyrimidine $E^{E^{\prime}}$ $+\mathrm{CO}_{2}\left(00^{0} 0\right) \rightarrow$ pyrimidine $^{E}+\mathrm{CO}_{2}\left(00^{0} 0, J, V\right)$ (the probability for energy transfer is given as $\operatorname{Prob}_{\mathrm{LJ}}=k_{2}^{J} / k_{\mathrm{LJ}}$, where $k_{\mathrm{LJ}}$ is the Lennard-Jones kinetic collision rate constant; it is defined (see Ref. 47) as $k_{\mathrm{LJ}}=\pi\left[\left(d_{\mathrm{CO}_{2}}\right.\right.$ $\left.\left.+d_{\text {pyrimidine }}\right) / 2\right]^{2} \sqrt{\left(8 k_{B} T\right) /(\pi \mu)} \Omega_{12}$, where $d_{\mathrm{CO}_{2}}=4.5 \AA$ is the $\mathrm{CO}_{2}$ cross section, Ref. $48, d_{\text {pyrimidine }}=5.27 \AA$ is the pyrimidine cross section (assumed to be equal to benzene, Ref. 48), $k_{B}$ is Boltzmann's constant, and $\mu$ is the reduced mass. $\Omega_{12}$ is the Lennard-Jones collision integral given by the following expression (Ref. 39): $\Omega_{12}=\left[0.636+0567 \log \left(k T / \varepsilon_{12}\right)\right]^{-1}$, where $\varepsilon_{12}$ is the pyrimidine- $\mathrm{CO}_{2}$ well depth with $\varepsilon_{\mathrm{CO}_{2}} / k=195 \mathrm{~K}$, Ref. $48, \varepsilon_{\text {pyrimidine }} / k$ $=456 \mathrm{~K}$ (determined by $1.15 T_{b}$ according to Ref. 48), and $\varepsilon_{12}=\sqrt{\varepsilon_{\mathrm{CO}_{2}} \varepsilon_{\text {pyrimidine }}}$; the Lennard-Jones collision rate constant at $298 \mathrm{~K}$ is $6.47 \times 10^{-10} \mathrm{~cm}^{3}$ molecule $\mathrm{s}^{-1}$.

\begin{tabular}{ccc}
\hline \hline Final $\mathrm{CO}_{2}$ rotational level & & \\
$J_{\text {final }}$ & $10^{12} \times k_{2}^{J}$ & $10^{3} \times \operatorname{Prob}_{\mathrm{LJ}}$ \\
\hline 58 & $8.4 \pm 2.1$ & $13 \pm 3$ \\
64 & $6.8 \pm 1.7$ & $11 \pm 3$ \\
66 & $6.7 \pm 1.7$ & $10 \pm 3$ \\
70 & $5.7 \pm 1.4$ & $8.7 \pm 2.2$ \\
72 & $4.9 \pm 1.2$ & $7.5 \pm 1.9$ \\
74 & $4.3 \pm 1.1$ & $6.7 \pm 1.7$ \\
76 & $4.3 \pm 1.1$ & $6.7 \pm 1.7$ \\
78 & $3.8 \pm 1.0$ & $5.9 \pm 1.5$ \\
80 & $3.5 \pm 0.9$ & $5.3 \pm 1.3$ \\
\hline \hline
\end{tabular}

determined from the UV absorption, thus allowing a determination of the rate constant $k_{2}^{J}$. Although Eq. (3) ignores the possibility that pyrimidine ${ }^{E^{\prime}}$ molecules, having undergone one collision, will collide a second time with $\mathrm{CO}_{2}$ again producing $\mathrm{CO}_{2}\left(00^{0} 0 ; \mathrm{J}, \mathrm{V}\right)$, a detailed kinetic analysis described elsewhere ${ }^{35}$ has been used to determine that the contribution of "second collisions" to the signal is at most $7 \%$ $12.5 \%$.

The absolute rate constants for the excitation of $\mathrm{CO}_{2}$ into the high $J$ tail of the ground vibrational level by collisions with hot pyrimidine $\left(E^{\prime}=40635 \mathrm{~cm}^{-1}\right)$ are given in Table II. As noted in the experimental description, these rate constants are scaled both on an absolute scale as well as to previous pyrazine $/ \mathrm{CO}_{2}$ scattering studies. ${ }^{10}$ Table II also includes the Lennard-Jones scattering probabilities for excitation to each final rotational state. The probability that a $\mathrm{CO}_{2}$ molecule is scattered into a particular final $J$ state is defined as the energy transfer rate constant, $k_{2}^{J}$, divided by the Lennard-Jones collision rate constant,

$$
\operatorname{Prob}^{J}=\frac{k_{2}^{J}}{k_{\mathrm{LJ}}} .
$$

Collisions populating these high $J$ states occur with frequencies similar to those measured in previously studied systems $;^{8-10,12}$ the probability for scattering into $J=58$ is $1.3 \times 10^{-2}$, corresponding to 1 out of 77 collisions exciting a molecule into the $\left(00^{0} 0 ; J=58\right)$ state. The probability for exciting $J=80$ is $5.3 \times 10^{-3}$, corresponding to 1 out of every 189 collisions resulting in a $\left(00^{\circ} 0 ; J=80\right) \mathrm{CO}_{2}$ molecule. The choice of the collision model (hard sphere or Lennard Jones) has an effect on both the magnitude of the energy transfer probability and the average amount of energy transferred per collision. The Lennard-Jones model is a more con- servative estimate of the collision rate and the resulting probabilities are therefore more accurate than those resulting from the use of a hard sphere collision rate. Lennard-Jones rate constants have been shown to be in error by as much as a factor of 2 for relaxation by collisions with HOD; ${ }^{36}$ however, it is yet to be tested whether Lennard-Jones rate constants are accurate for $\mathrm{CO}_{2}$. Despite potential errors, use of Lennard-Jones rate constants allows direct comparison of energy transfer probabilities from system to system, since $k_{\mathrm{LJ}}$ were used for previously studied systems.

\section{Energy transfer probability distribution function}

The energy transfer probability distribution function, $P\left(E, E^{\prime}\right)$, for the large $\Delta E$ region can be obtained from state resolved data reported here; the method for this conversion process has been detailed elsewhere. ${ }^{7}$ Although the results reported here give a very detailed picture of postcollision $\mathrm{CO}_{2}$ molecules, the initial states can only be described by a room temperature distribution. To overcome this limitation, an average initial rotational state and translational velocity have been used. For pyrazine, ${ }^{10}$ perfluorobenzene, ${ }^{8}$ and methylpyrazine ${ }^{9}$ collisions with $\mathrm{CO}_{2}$, detailed temperature dependent measurements of the energy transfer process, along with an angular momentum gap law model, were used to provide the average initial rotational state, $\left\langle J_{i}\right\rangle$. For these systems, the average was determined to be $\left\langle J_{i}\right\rangle=26.5,28.7$, and 31 for methylpyrazine, pyrazine, and $\mathrm{C}_{6} \mathrm{~F}_{6}$, respectively. Because the difference in rotational energy between the initial and final states for a linear molecule goes as the difference in $J^{2}$, uncertainty in the final state produces a greater error than does uncertainty in the initial state. Assuming an average initial rotational state of $\left\langle J_{i}\right\rangle=28$ for methylpyrazine and $\mathrm{C}_{6} \mathrm{~F}_{6}$ results in an error of $2.2 \%$ and $5.1 \%$ for $J_{f}=82$ and 58 , respectively. This error is about the same or smaller than other experimental errors associated with these studies. Because the error is relatively small for making an assumption about the initial state, temperature dependent studies have not been performed for the pyrimidine $/ \mathrm{CO}_{2}$ system. The average initial rotational state determined in pyrazine $/ \mathrm{CO}_{2}$ (Ref. 10) 248-nm studies $\left(\left\langle J_{i}\right\rangle=28.7\right)$ has been used to calculate $P\left(E, E^{\prime}\right)$. Unlike the average initial $J$, temperature dependent studies are not necessary to determine the average initial relative velocity; these have been calculated as before using a translational gap law model. ${ }^{10}$

The energy transfer distribution function for pyrimidine relaxation by single carbon dioxide collisions is plotted in Fig. 5. (Note that the probability is plotted against $E-E^{\prime}$ where $E^{\prime}=40635 \mathrm{~cm}^{-1}$ and $E$ is the final donor energy.) A comparison of an energy transfer distribution function obtained from energy transfer data extrapolated over the full range of $\Delta E$ with the function calculated just from the experimental data suggests that $P\left(E, E^{\prime}\right)$ in the energy range $>1300 \mathrm{~cm}^{-1}$ (shown in Fig. 5) is well determined in these experiments. A principal aspect of this work is to determine how molecular properties affect the energy transfer probability distribution function; thus, Fig. 5 also shows $P\left(E, E^{\prime}\right)$ distributions for pyrazine, ${ }^{7}$ perfluororbenzne, ${ }^{7}$ and methylpyrazine ${ }^{9}$ excited to similar energies for comparison 


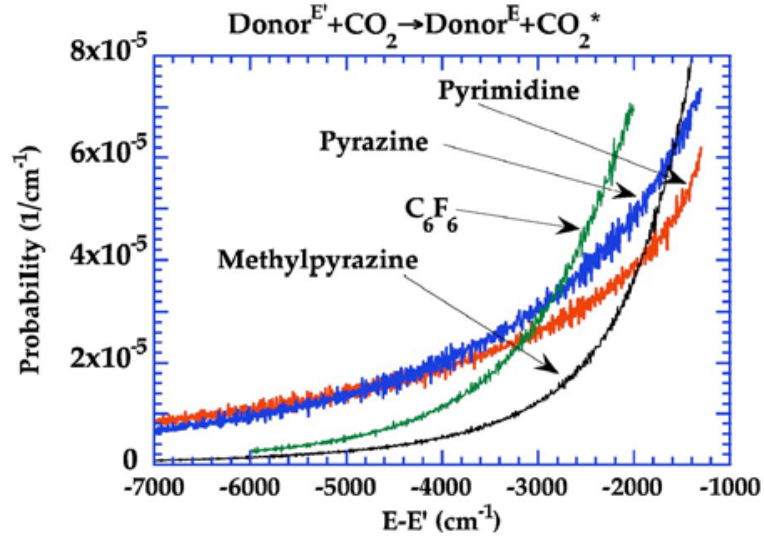

FIG. 5. (Color online) Shown are plots of the high $\Delta E$ tail of the energy transfer probability distribution function, $P\left(E, E^{\prime}\right)$, describing the first Lennard-Jones collision between a vibrationally excited donor molecule [pyrimidine, pyrazine, (Ref. 10), $\mathrm{C}_{6} \mathrm{~F}_{6}$, (Ref. 8), or methylpyrazine (Ref. 9)], at energy $E^{\prime} \approx 41000 \mathrm{~cm}^{-1}$ and a $\mathrm{CO}_{2}$ bath molecule resulting in $\mathrm{CO}_{2}$ excitation into high rotational angular momentum states $(J=58-82)$ of the ground vibrational level. $E$ is the energy of the hot donor molecule following the collision.

with the current work. There have been a number of bathprobe energy transfer studies performed at other excitation energies (for example, $\lambda=251-324 \mathrm{~nm}$ ) ${ }^{13-16,37}$ not surprisingly, these studies indicate that energy transfer is dependent on the precollision energy of the donor molecule. Because of this dependence, the majority of comparisons made to the present work will be limited to other studies performed at $\lambda=248 \mathrm{~nm}$. For all donor molecules, single collisions with $\Delta E \geqslant 5000 \mathrm{~cm}^{-1}$ are clearly evident; however, this supercollision tail behaves differently for each molecule. Considering similarities in molecular weight, structure, and vibrational modes, it is not surprising that $P\left(E, E^{\prime}\right)$ for pyrimidine is similar in shape and magnitude to the distribution function for pyrazine; however, both have a different shape than $\mathrm{C}_{6} \mathrm{~F}_{6}$ and methylpyrazine in this $\Delta E$ region. For example, at $\Delta E$ $=5000 \mathrm{~cm}^{-1} P\left(E, E^{\prime}\right)$ decreases noticeably going from pyrazine and pyrimidine to hexafluorobenzene to methylpyrazine. On the other hand, the pyrazine and pyrimidine $P\left(E, E^{\prime}\right)$ 's cross the curve for $\mathrm{C}_{6} \mathrm{~F}_{6}$ near $\Delta E=3000 \mathrm{~cm}^{-1}$ and the curve for methylpyrazine near $\Delta E=2000 \mathrm{~cm}^{-1}$. Since $P\left(E, E^{\prime}\right)$ is a normalized distribution, these shifts in amplitude versus $\Delta E$ show that the probability distribution changes from molecule to molecule. Also because of normalization, curves must cross one another at some value of $\Delta E$, or be the same; thus, any comparison between $P\left(E, E^{\prime}\right)$ for different systems requires specification of both final and initial energies. As a result it is convenient to fit $P\left(E, E^{\prime}\right)$ to a model function, which allows one to make comparisons between systems using only one or two parameters.

To facilitate comparison between these different systems, the energy transfer distribution function shown in Fig. 5 can be fitted to either a single or a biexponential functional form. Single exponential fitting of $P\left(E, E^{\prime}\right)$ obtained in previous studies has been shown to fit the experimentally obtained distribution function poorly, when normalization and detailed balance are considered; however, for pyrazine and $\mathrm{C}_{6} \mathrm{~F}_{6}{ }^{7}$ the average energy transferred in down collisions, $\langle\Delta E\rangle_{d}$ obtained from the single exponential fit compared well to donor energy loss studies, and is used here for comparison. The normalized, biexponential model, which has been used to include both strong and weak collisions, accurately fits the data within the restrictions of normalization and detailed balance. This function is given according to ${ }^{38}$

$$
\begin{aligned}
& P\left(E, E^{\prime}\right)=\frac{(1-f) \exp \left\{-\left(E^{\prime}-E\right) / \alpha\right\}+f \exp \left\{-\left(E^{\prime}-E\right) / \gamma\right\}}{(1-f)(\alpha+\beta)+f(\gamma+\delta)}, \quad E \leqslant E^{\prime}, \\
& P\left(E, E^{\prime}\right)=\frac{(1-f) \exp \left\{-\left(E-E^{\prime}\right) / \beta\right\}+f \exp \left\{-\left(E-E^{\prime}\right) / \delta\right\}}{(1-f)(\alpha+\beta)+f(\gamma+\delta)}, \quad E>E^{\prime} .
\end{aligned}
$$

In this model $\alpha$ is the average energy transfer for downward weak collisions, while $\gamma$ is the average energy transfer for downward strong (super) collisions. $\beta$ and $\delta$ are the respective average energy transfers for the upward weak and strong collisions and are related to $\alpha$ and $\gamma$ by detailed balance ${ }^{39}$ according to the expressions

$$
\begin{aligned}
& \beta=\frac{\alpha F_{E} k T}{\alpha+F_{E} k T}, \\
& \delta=\frac{\gamma F_{E} k T}{\gamma+F_{E} k T} .
\end{aligned}
$$

$F_{E}=1.117$ is a quantity related to the energy dependence of the donor density of states $\mathrm{s}^{39,40}$ and is calculated according to an approximation derived by Troe. ${ }^{41}$ The denominators in Eqs. (5a) and (5b) ensure that $P\left(E, E^{\prime}\right)$ is normalized. Note that the separation of collisions into weak and strong channels is somewhat arbitrary; the biexponential model is used in systems where the large $\Delta E$ tail of $P\left(E, E^{\prime}\right)$ is larger than would be predicted by a single exponential model. Although $f$ should not be interpreted literally as the fraction of supercollisions since this channel is peaked at $\Delta E=0 \mathrm{~cm}^{-1}$, the fraction of large $\Delta E$ collisions does get larger with increasing $f$. The relative importance of supercollisions in $P\left(E, E^{\prime}\right)$ depends on the magnitude of both $f$ and $\gamma$. The energy transfer distribution along with the best-fit, biexponential model function [Eqs. (5a) and (5b)] is shown in Fig. 6 for the pyrimidine/ $\mathrm{CO}_{2}$ system along with the parameters that define 


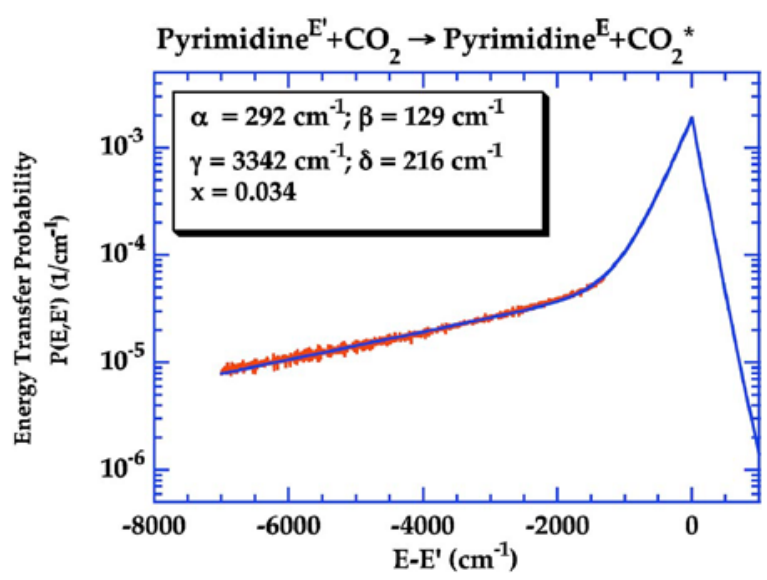

FIG. 6. (Color online) Plot of the energy transfer distribution function for the pyrimidine $/ \mathrm{CO}_{2}$ system with $E^{\prime}=40635 \mathrm{~cm}^{-1}$ (noisy line), along with the best-fit biexponential model function [Eqs. (5a) and (5b)] (solid line). The best-fit parameters $(\alpha, \beta, \gamma, \delta$, and $f)$ are defined in the text following Eqs. (5a) and (5b). $E$ is the energy of the hot donor molecule following the collision. The $P\left(E, E^{\prime}\right)$ shown is calculated based on the estimated values of $\left\langle J_{i}\right\rangle=28.7$ for the mean initial rotational state for collisions populating high $J_{f}$ states $\left(J_{f} \approx 58-80\right)$ of $\mathrm{CO}_{2}$.

the best fit to the data using this model. Figure 6 shows that the strong-collision tail of the distribution is clearly exponential; however, if this exponent were used to extrapolate the remainder of $P\left(E, E^{\prime}\right)$, the total probability would be significantly less than 1 . This clearly illustrates that $P\left(E, E^{\prime}\right)$ for the pyrimidine $/ \mathrm{CO}_{2}$ system must be a composite of at least two functions. It should be pointed out that Eqs. (5a) and (5b) are not five-parameter fits, even though there are five different variables that define the function, since two are fixed by the detailed balance relationship. Of the three remaining, one is well defined by the experimental results - the strong-collision parameter, $\gamma$. The fraction of the strongcollision exponent is also well defined by the magnitude of the energy transfer rate probabilities, but not as well as the shape of this part of the curve since its accuracy depends on the accuracy of the Lennard-Jones collision rate. This means that the remaining function is then fixed by the requirements of normalization. The biexponential parameters are similar in value to those found in previous energy transfer studies, but as expected they are most similar to those of pyrazine. ${ }^{7}$

\section{E. Energy transfer trends}

One of the objects of this work is to obtain a greater understanding of the molecular features responsible for large $V \rightarrow R T$ energy transfer events in the quenching of highly vibrationally excited donor molecules in collisions with $\mathrm{CO}_{2}$. In particular, we are interested in increasing our understanding of the factors that affect the shape and magnitude of $P\left(E, E^{\prime}\right)$.

The same general dynamic features have been observed in these experiments as have been observed for previously studied systems. Large amounts of pyrimidine vibrational energy are transferred to $\mathrm{CO}_{2}$, which becomes both rotationally and translationally excited. This similarity is further seen in the fact that $P\left(E, E^{\prime}\right)$ for the pyrimidine $/ \mathrm{CO}_{2}$ is similar in magnitude to those previously observed. As noted above, in all cases studied thus far, a single exponential form is not sufficient to fit the data, given the constraints of normalization and detailed balance; thus a normalized, biexponential model, which includes super or strong collisions as well as weak collisions in the energy transfer distribution, has been used. Although other $P\left(E, E^{\prime}\right)$ model functions have been successfully used by others, the biexponential model has been chosen for several reasons. First, the experimentally derived tail of $P\left(E, E^{\prime}\right)$ is clearly exponential, as seen in Fig. 6 ; thus, some type of exponential function is appropriate for these data. The next simplest model function results from adding a second exponential function to the first. Some have successfully used a stretched exponential function to fit experimentally measured $P\left(E, E^{\prime}\right)$ functions; ${ }^{5}$ however, this is not necessary here since a biexponential function accurately fits the experimental data within the system constraints of normalization and detailed balance. Additionally, the upward turn in the data near $1500 \mathrm{~cm}^{-1}$ indicates that the shape of $P\left(E, E^{\prime}\right)$ is changing from an exponential function to some other function, likely a different exponential.

As mentioned before, any comparison of $P\left(E, E^{\prime}\right)$ between different donors without a model function requires a specification of $E$ and $E^{\prime}$ to be meaningful; however, the biexponential fit provides several parameters for comparison. Table III lists the various biexponential fit parameters for systems studied at $248 \mathrm{~nm}$ to date. $\mathrm{C}_{6} \mathrm{~F}_{6}$ has the largest fraction of strong collisions followed by methylpyrazine, pyrazine, and pyrimidine, with values of $f=0.15,0.05,0.05$, and 0.03 , respectively. The characteristic energies of both the weak $(\alpha)$ and strong $(\gamma)$ collisions are also of interest. The characteristic energies of the strong collisions for $\mathrm{C}_{6} \mathrm{~F}_{6}$, methylpyrazine, pyrazine, and pyrimidine are $\gamma=1411,1527$, 2532 , and $3342 \mathrm{~cm}^{-1}$, respectively, while the values for weak collisions are $\alpha=620,441,296$, and $292 \mathrm{~cm}^{-1}$, respectively. Although $\mathrm{C}_{6} \mathrm{~F}_{6}$ has the largest fraction of supercollisions $(f$ $=0.15$ compared to 0.05 for pyrazine and methylpyrazine and 0.03 for pyrimidine), these supercollisions are not as super on average as those for the other three molecules based on their respective characteristic energies for strong collisions. However, as the values for $\alpha$ and $\gamma$ become closer to one another, the biexponential fit approaches a single exponential; therefore, the distinction between weak and strong collisions for $\gamma \sim \alpha$ is not as significant.

Table III also includes various molecular properties for the different donor molecules, such as the number of vibrational modes, the number of modes with a frequency less than $500 \mathrm{~cm}^{-1}$, donor density of states, and dissociation lifetime. Trends observed in the biexponential fit parameters seem to be consistent with trends in these properties as well as the average vibrational energy per mode. ' Table III is tabulated by increasing strong-collision energies $(\gamma)$, which generally correspond to decreasing average energy transferred in downward collisions $\left(\langle\Delta E\rangle_{d}\right)$, weak-collision value $(\alpha)$, fraction of strong collisions $(f)$, density of states $\left[\rho\left(E^{\prime}\right)\right]$, dissociation lifetime $\left(\tau_{\text {dis }}\right)$, number of vibrational modes $(s)$, and number of modes with $\nu<500 \mathrm{~cm}^{-1}$.

A comparison of the average energy transferred in down collisions, reported in Table III, shows that $\langle\Delta E\rangle_{d}$ is generally smaller for donors with fewer vibrational modes. How- 
TABLE III. Energy transfer probability distribution function double exponential fit parameters (the characteristic energy transfer frequencies for both strong and weak down collisions, the fraction of strong collisions, and the average down collision energy) as well as the total number of donor vibrational modes, the number of low-frequency vibrational modes, the precollision density of states, and the photodissociation lifetime.

\begin{tabular}{|c|c|c|c|c|c|c|c|c|}
\hline & $\begin{array}{c}\gamma \\
\left(\mathrm{cm}^{-1}\right)^{\mathrm{a}}\end{array}$ & $\begin{array}{l}\langle\Delta E\rangle_{d}{ }^{\mathrm{b}} \\
\left(\mathrm{cm}^{-1}\right)\end{array}$ & $\begin{array}{c}\alpha \\
\left(\mathrm{cm}^{-1}\right)^{\mathrm{c}}\end{array}$ & $f^{\mathrm{d}}$ & $s^{\mathrm{e}}$ & $\rho\left(E^{\prime}\right)^{\mathrm{f}}$ & $\begin{array}{l}\tau_{\mathrm{dis}}{ }^{\mathrm{g}} \\
(\mu \mathrm{s})\end{array}$ & $s<500 \mathrm{~cm}^{-1 \mathrm{~h}}$ \\
\hline $\mathrm{C}_{6} \mathrm{~F}_{6}{ }^{\mathrm{i}}$ & 1411 & 809 & 620 & 0.15 & 30 & $2.1 \times 10^{23}$ & $\infty$ & 13 \\
\hline Methylpyrazine $e^{j}$ & 1527 & 682 & 441 & 0.05 & 33 & $8.4 \times 10^{17}$ & $\sim 16$ & 4 \\
\hline Pyrazine $^{\mathrm{k}}$ & 2532 & 536 & 296 & 0.05 & 24 & $3.9 \times 10^{13}$ & $\sim 6$ & 2 \\
\hline Pyrimidine & 3342 & 576 & 292 & 0.03 & 24 & $1.8 \times 10^{13}$ & $\sim 1$ & 2 \\
\hline
\end{tabular}

${ }^{\mathrm{a}}$ The characteristic strong energy transfer frequency as determined from the biexponential fit of the $P\left(E, E^{\prime}\right)$ data.

${ }^{\mathrm{b}}$ The average energy transferred in a single downward collision involving vibrationally excited donor and $\mathrm{CO}_{2}$. Calculated by fitting $P\left(E, E^{\prime}\right)$ to an exponential model. See Refs. 7-9 for details.

${ }^{c}$ The characteristic weak energy transfer frequency as determined from the biexponential fit of the $P\left(E, E^{\prime}\right)$ data.

${ }^{\mathrm{d}}$ The fraction of strong collisions as determined from the biexponential fit of the $P\left(E, E^{\prime}\right)$ data.

${ }^{\mathrm{e}}$ The total number vibrational normal modes for the donor molecule.

${ }^{\mathrm{f}}$ The vibrational density of states for the donor molecule at the energy following the absorption of a 248 -nm photon calculated using a Whitten-Rabinovitch algorithm (Ref. 49).

${ }^{\mathrm{g}}$ The photodissociative lifetime of the donor molecule after 248-nm absorption. Lifetimes for $\mathrm{C}_{6} \mathrm{~F}_{6}$, methylpyrazine, pyrazine, and pyrimidine are obtained from Refs. 8, 50, 27 and 28, and 26 respectively.

${ }^{\mathrm{h}}$ The number of vibrational normal modes with frequency less than $500 \mathrm{~cm}^{-1}$. Normal mode frequencies for $\mathrm{C}_{6} \mathrm{~F}_{6}$, methylpyrazine, pyrazine, and pyrimidine are obtained from Refs. 51, 52, 53, and 54, respectively.

${ }^{1} P\left(E, E^{\prime}\right)$ parameters obtained from Ref. 7

${ }^{\mathrm{j}} P\left(E, E^{\prime}\right)$ parameters obtained from Ref. 9 .

${ }^{\mathrm{k}} P\left(E, E^{\prime}\right)$ parameters obtained from Ref. 7.

ever, the average for the entire distribution is heavily weighted by small $\Delta E$ events that are most probable; thus, the average alone provides little insight into either $P\left(E, E^{\prime}\right)$ shape or to factors that influence it. On the other hand, the strong- and weak-collision energies provide direct information about the shape of $P\left(E, E^{\prime}\right)$. The parameter describing the shape of the strong-collision energy transfer region, $\gamma$, increases with decreasing number of donor vibrational modes, while the parameter describing the shape of the weak-collision energy transfer region, $\alpha$, increases with increasing number of donor modes. In simple terms, this can be understood by the fact that if energy is constant ( $E^{\prime}$ $\sim 41000 \mathrm{~cm}^{-1}$ for each donor in these studies) as the number of vibrational modes increases, the amount of energy per mode decreases. Thus there is less energy per mode on average as the number of vibrational modes becomes greater, which reduces the probability of large $\Delta E$ events and increases the probability of small $\Delta E$ events, since $P\left(E, E^{\prime}\right)$ is normalized. This simple qualitative argument assumes that the vibrational frequencies of the various donor modes are essentially the same.

A more accurate picture of how internal energy is distributed amongst the donor vibrational modes can further aid in understanding the relationship between $P\left(E, E^{\prime}\right)$ shape and number of donor modes. The average vibrational energy per mode, $\left\langle E_{\mathrm{vib}}\right\rangle_{i}$ for each donor molecule can be calculated using $^{42}$

$$
\left\langle E_{\mathrm{vib}}\right\rangle_{i}=\frac{h \nu_{i} \sum_{v=1}^{\max } v \rho_{s-1}\left(E-v h \nu_{i}\right)}{\rho_{s}(E)},
$$

where $\rho_{s}(E)$ and $\rho_{s-1}\left(E-v h \nu_{i}\right)$ are the density of states for all $s$ oscillators at energy $E$, and the density of states for all oscillators except the mode of interest at an energy of $E$ $-v h \nu_{i} . \nu_{i}$ is the frequency of the mode of interest and $v$ is the vibrational quantum number of that mode, with $v=\max$ corresponding to the maximum possible value of the vibrational quantum number of that mode, given according to max $=E / h \nu_{i}$. The average vibrational energy per mode as a function of vibrational mode frequency is plotted in Fig. 7 for each donor molecule. As expected, for each donor the lowest-frequency mode contains the greatest amount of energy and hence the greatest number of quanta. Pyrazine and pyrimidine have both the highest-frequency modes as well as the fewest number of modes; therefore, as predicted their curves are the highest. Methylpyrazine with the most modes might be expected to be the lowest curve; however, $\mathrm{C}_{6} \mathrm{~F}_{6}$ has a large number of modes with low frequency ( 15 with a frequency less than $500 \mathrm{~cm}^{-1}$ ) which changes the order of these two curves from what might be predicted based solely on the number of vibrational modes. Although the simple model of more modes corresponding to less energy per mode

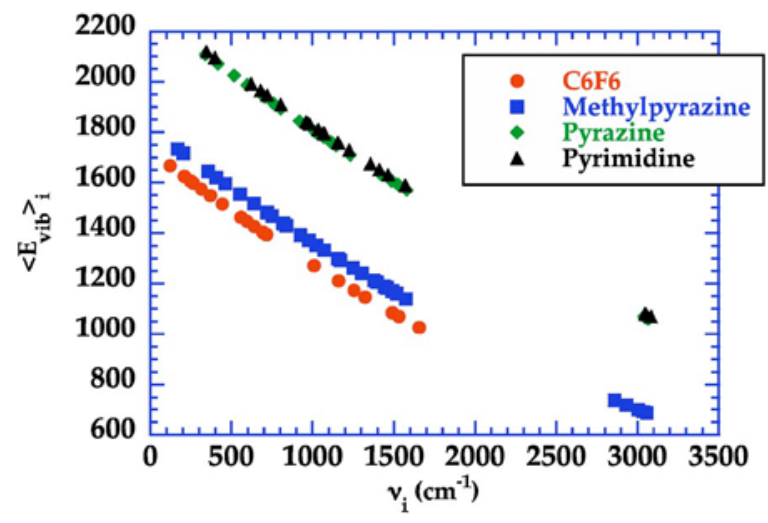

FIG. 7. (Color online) The average vibrational energy of the individual vibrational modes $\left(\left\langle E_{\mathrm{vib}}\right\rangle_{i}\right)$ for highly vibrationally excited $\mathrm{C}_{6} \mathrm{~F}_{6}$, methylpyrazine, pyrazine, and pyrimidine plotted as a function of vibrational frequency $\left(\nu_{i}\right)$. Not surprisingly the average vibrational energy per mode, calculated using Eq. (8) is similar for pyrazine and pyrimidine. 
and therefore a smaller energy transfer is generally true, as seen with these systems, it is necessary to also consider the frequency of those modes.

Although there appears to a be simple correlation between the number of modes, the average energy per mode, and the average energy transfer when considering the tail, this correlation may be somewhat misleading. These correlations suggest that large energy transfer is maximized for stiff high-frequency modes, yet the average energy transferred in down collisions tabulated in Table III suggests just the opposite; those results indicate that low-frequency modes lead to large average energy transfers.

Both quantum-scattering ${ }^{21}$ calculations by Clary et al. and classical trajectory ${ }^{20,43}$ calculations by Lendvay and coworker indicate that low-frequency vibrational modes in the donor molecule have large energy transfer cross sections, while high-frequency modes have small cross sections. At first blush this may seem counter to the pyrazine and pyrimidine data with their large values of $\gamma$ however, energy transfer processes are more complex than can be summed up in a single term. Table III shows that there is a direct correlation between the number of low frequency vibrations, the density of states, and the fraction of strong collisions. If the works of Clary et al. and Lendvay and co-worker can be used to accurately interpret the results presented here, then perhaps low-frequency modes give rise to the strong collisions, but do not directly govern their magnitude, [i.e., the shape of $\left.P\left(E, E^{\prime}\right)\right] . \mathrm{We}^{44}$ have recently calculated $P\left(E, E^{\prime}\right)$ from previously published energy transfer data for pyrazine energy transfer as a function of internal energy. ${ }^{37}$ According to our calculations, the fraction of strong collisions increases as the donor density of states increases, while the shape of the $P\left(E, E^{\prime}\right)$ (determined by $\alpha$ and $\gamma$ ) is constant over certain energy regions. This supports the calculations of Clary et al. and Lendvay and co-worker. Thus it seems that both the number of vibrational modes and their frequency are important in determining the overall nature of the energy transfer process and thus the shape of $P\left(E, E^{\prime}\right)$.

The results of the vibrational close-coupling, infinite order sudden approximation quantum-scattering calculations by Clary et al. further indicate that in addition to the importance of donor mode frequency, the motion of the mode is also important in determining the efficiency of energy transfer events. Even when the frequency of the in-plane vibrational mode with the greatest energy transfer efficiency is artificially altered to be the same as the most efficient outof-plane mode, the energy transfer efficiency of the out-ofplane mode is still greater. Lendvay, ${ }^{20}$ in his classical trajectory study of collisional energy transfer from vibrationally excited $\mathrm{CS}_{2}$, made the same observation-both frequency and mode character are responsible for efficient collision energy transfer. He referred to the modes involved in energy transfer as "gateways" or "conduits" through which energy "leaks out" of the donor into the bath molecule. Nongateway modes only become active in the energy transfer process when their vibrational frequency is artificially lowered and the vibrational frequency of the gateway modes is increased. Simply lowering the frequency of the nongateway modes does not make them as efficient as the gateway modes. Thus in addition to the number of modes and their frequency, their motion is an important consideration in understanding the energy transfer process and the shape of $P\left(E, E^{\prime}\right)$. We have just completed a study ${ }^{34}$ of energy transfer using the three difluororbenzene isomers as energy transfer donors. This study is intended to isolate the effect of vibrational mode character on the energy transfer process.

It is important to bear in mind that the results of Lendvay and co-worker and Clary et al. were carried out either with smaller donor molecules or smaller amounts of internal energy or both; therefore, their findings might not extrapolate to the systems and energies in these experiments. That said, it is clear that mode character and frequency must be considered for their effect on the shape and magnitude of $P\left(E, E^{\prime}\right)$. A model that can be used to determine the effect of various donor vibrational modes on the shape of $P\left(E, E^{\prime}\right)$ has been developed elsewhere. ${ }^{9}$ This model function

$$
f\left(E, E^{\prime}\right)=(\text { efficiency factor }) \times \sum_{v=m}^{\max } f_{v_{i}}
$$

is determined by two factors: first, the efficiency of a given vibrational mode at transferring energy, and second, the ability of the mode to transfer energy. A given vibrational mode $\nu_{\mathrm{i}}$ may be a very efficient energy transfer donor, but if there are only a few donor molecules in that vibrational mode with $v \cdot h \nu_{i}=\Delta E$, its contribution to $P\left(E, E^{\prime}\right)$ will be small despite its large energy transfer efficiency. The converse is also true. A mode that is a very poor donor may still make a significant contribution to $P\left(E, E^{\prime}\right)$ if the probability of finding a large number of molecules with $\Delta E=v \cdot h \nu_{i}$ of energy in that mode is large enough. These two factors, the efficiency of the donor mode as well as the probability of finding a large enough amount of energy in that mode, both affect $P\left(E, E^{\prime}\right)$.

Ability to transfer energy is determined by the probability that a donor occupies a given vibrational mode $\nu_{i}$ with sufficient energy to cause the energy transfer $\Delta E$. This is calculated according to

$$
f_{v_{i}}=G_{i} \frac{\rho_{s-1}\left(E^{\prime}-v h \nu_{i}\right)}{\rho_{s}\left(E^{\prime}\right)},
$$

where $G_{i}$ accounts for degeneracy of the $i$ th mode. This fraction is then summed from the minimum number of quanta, $m$, necessary to impart a given $\Delta E$, such that $m h \nu_{i}=\Delta E$, to the maximum number of quanta possible for that mode. Relative energy transfer efficiency of a given mode is determined from the experimentally measured $P\left(E, E^{\prime}\right)$ shown in Fig. 5. Energy transfer probability is equal at points were $P\left(E, E^{\prime}\right)$ intersect for different systems. Assuming that one donor mode dominates that energy transfer process, it is straightforward to calculate an efficiency factor, ${ }^{9}$ which is then applied to all other values of $\Delta E$.

Assuming energy transfer events that populate the large $\Delta E$ portion of $P\left(E, E^{\prime}\right)$ result entirely from collisions from $\nu_{16}$, a low-frequency out-of-plane vibrational mode observed to have the largest energy transfer cross section in the quantum-scattering calculations of Clary et al., ${ }^{21}$ and using the $f\left(E, E^{\prime}\right)$ model produces a set of curves that more closely mirror the shape of the experimental $P\left(E, E^{\prime}\right)$ 's, than do the 


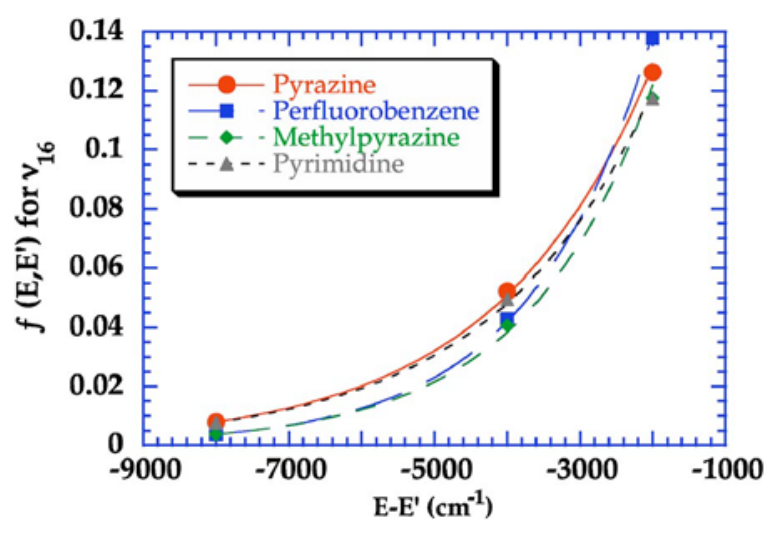

FIG. 8. (Color online) The $f\left(E, E^{\prime}\right)$ function (fractional mode populations times an efficiency factor) for the $\nu_{16}$ out-of-plane mode for pyrimidine, pyrazine, methylpyrazine, and $\mathrm{C}_{6} \mathrm{~F}_{6}$ as a function of $\Delta E=E-E^{\prime}\left(\mathrm{cm}^{-1}\right)$. The fractional mode populations are calculated using Eq. (10) and have been scaled according to the relative energy transfer efficiency determined from the points where $P\left(E, E^{\prime}\right)$ have equal energy transfer probability for different donors as seen in Fig. 5.

set of $f\left(E, E^{\prime}\right)$ curves created by assuming energy transfer is due entirely to $\nu_{2}$, a high-frequency in-plane mode. These curves, displayed in Fig. 8, support the conclusion that the motion of the donor vibrational mode also influences $P\left(E, E^{\prime}\right)$ shape. One caveat must be realized in this model: it is only possible to determine relative efficiency of the same molecule motion between different donors, and it is not possible to determine the relative efficiency of different modes within the same donor molecule.

Another factor to be considered in the shape of $P\left(E, E^{\prime}\right)$ is the proximity of the donor molecule to its dissociation threshold. As seen in Table III, the magnitude of the strongcollision energy increases as the donor gets closer to its dissociation threshold as indicated by lifetime. Trajectory calculations ${ }^{18}$ of relaxation of highly excited $\mathrm{SO}_{2}$ by $\mathrm{Ar}$ show that energy transfer efficiency increases dramatically approaching the reaction threshold. Mullin and co-workers have observed that the energy transfer rate constants for pyrazine $/ \mathrm{CO}_{2}$ collisions also increase dramatically as the amount of internal energy approaches the pyrazine threshold. ${ }^{37}$ Based on this, it seems possible that although the number of strong collisions and to some degree the shape of $P\left(E, E^{\prime}\right)$ are governed by mode frequency and motion, the magnitude of the strong collision may be related to the proximity of the donor molecule to its dissociation energy. In other words, pyrimidine may not have as many supercollisions as $\mathrm{C}_{6} \mathrm{~F}_{6}$, but they are on average more super, because at $E^{\prime} \sim 41000 \mathrm{~cm}^{-1}$ pyrimidine is about ready to explode in a unimolecular process. "Superexcited" pyrimidine molecules on the verge of breaking apart undergo large amplitude anharmonic motion that is likely to affect the magnitude of the supercollisions. Photodissociation studies performed in our laboratory ${ }^{45}$ indicate that the motions leading to dissociation are the same motions responsible for large energy transfer processes. Thus as a molecule approaches threshold, the energy transfer modes (the motions that also lead to dissociation) become more active.

Mullin and co-workers ${ }^{14,16}$ have suggested that time dependent perturbation theory can be used to accurately de- scribe the energy transfer events observed in collisions between highly vibrationally excited donor molecules and cold bath molecules. Initially, this may seem unreasonable since the use of perturbation theory requires only a small perturbation be applied to the system; however, the collision which provides the perturbation has a perturbing energy close to $E=\mathrm{kT} \sim 200 \mathrm{~cm}^{-1}$. Naturally, the greater the energy transfer magnitude, $\Delta E$, the greater the difference between the energy of the initial and final states, the smaller the probability of this energy transfer. Additionally, the perturbation only acts for a short time, since the energy loss occurs in the sudden limit of molecular collisions, in other words the collision time is short compared to the period of vibrational motion. ${ }^{46}$ Classical trajectory calculations by Clary et $a .^{21}$ show that the time of the energy transfer process (the "flick") is very short (40-60 fs) compared to the period of vibrational motion ( $\sim 0.1 \mathrm{ps}$ for low-frequency vibrational modes), even though duration of the collision may be several picoseconds. More recent simulations ${ }^{22}$ indicate that $\sim 50 \%$ of collisions between vibrationally excited pyrazine and $\mathrm{CO}_{2}$ that result in large $V \rightarrow$ RT energy transfer occur in multiple donor/bath encounters (i.e., multiple flicks) These multiple encounter collisions are referred to as "chattering collisions." Depending on the number of encounters involved in the chattering collision, the total chattering collision lifetime may be as long as several vibrational periods; however, typical pyrazine $/ \mathrm{CO}_{2}$ supercollision trajectories indicate that the time of the individual encounters is still short and that multiple impulsive encounters are separated by 300-700 fs. Using time dependent perturbation theory to calculate the rate of transition from a dense manifold of vibrational states to a less dense manifold of states one obtains a modified form of Fermi's golden rule, which states that $P\left(E, E^{\prime}\right)$ should be proportional to the density of donor states

$$
P\left(E, E^{\prime}\right) \propto\left|V_{i f}\right|^{2} \rho(E) \rho\left(E^{\prime}\right),
$$

where $\rho(E)$ is the density of states at energy $E$ and $V_{i f}$ is the matrix element, $\langle i|H| f\rangle$, that couples initial and final states. As pointed out by Mullin and co-workers ${ }^{14,16}$ the importance of Eq. (11) is that the shape of the probability distribution function should mirror the shape of the final donor density of states. Alternatively, the "slope" of $P\left(E, E^{\prime}\right)$ should be related to the slope of the final donor density of states as a function of $\Delta E$. Figure 9 shows a plot of the inverse of the characteristic energy transfer energies obtained by fitting $P\left(E, E^{\prime}\right)$ to Eqs. (5a) and $(5 b)$ as a function of the slope obtained from plotting $\operatorname{In}\left[\rho\left(E^{\prime}-\Delta E\right)\right]$ vs $\Delta E .{ }^{15}$ As can be readily seen, both the strong and weak characteristic energy transfer magnitudes are linearly related to the slope of $\ln [\rho(E-\Delta E)]$ vs $\Delta E$. The results fit remarkably well, especially considering that the weak-collision energy is obtained by extrapolation of the data. This correlation between density of states and $P\left(E, E^{\prime}\right)$ suggests that the donor density of states (which is governed primarily by the low-frequency modes) is largely responsible for the "shape" of $P\left(E, E^{\prime}\right)$. Additionally, this may provide a means to predict $P\left(E, E^{\prime}\right)$, at least in terms of its shape. Mullin and co-workers have shown that this relationship holds true for the shape of the tail of the distribution for other aromatic donors ${ }^{13-15}$ as well 


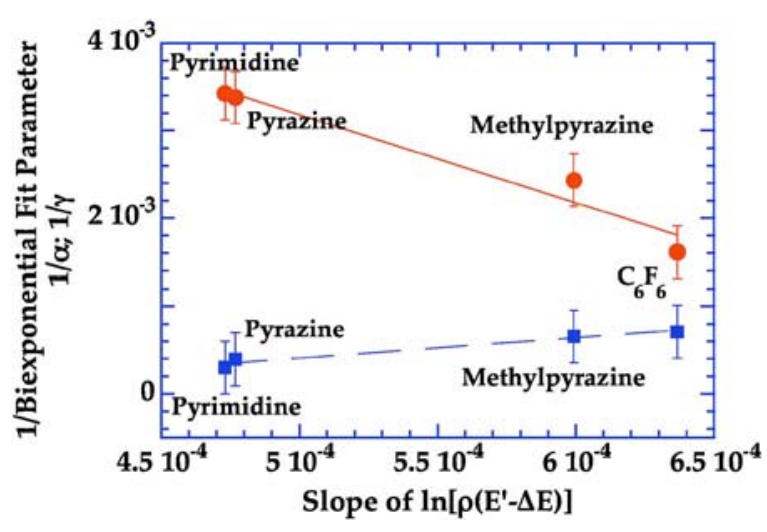

FIG. 9. (Color online) The correlation between the shape of $P\left(E, E^{\prime}\right)$ as determined by the biexponential fit parameters $(1 / \alpha$ and $1 / \gamma)$ and the energy dependent state density of the donor molecules. Squares represent the characteristic energy transfer magnitude for strong collisions $(\gamma)$ while the circles are the characteristic energy transfer magnitude for weak collisions $(\alpha)$. Based on the application of Fermi's golden rule to collisional deactivation of vibrationally excited donor molecules in collisions with $\mathrm{CO}_{2}$. Pyrazine, $\mathrm{C}_{6} \mathrm{~F}_{6}$, and methylpyrazine data are from Refs. 7-9. The shape of the energy transfer distribution function as a function of $\Delta E$ mirrors the shape of the final donor density of states as a function of energy transferred $(\Delta E)$. The shape of both the strong- and weak-collision regions of $P\left(E, E^{\prime}\right)$ is correlated with the donor molecule's final density of states.

as for collisional deactivation by collisions with water. ${ }^{16}$ However, because the proportionality constant is not known for Eq. (11) and the average energy transfer energy of the weak collisions is obtained through an extrapolated fit of the data, the overall magnitude of $P\left(E, E^{\prime}\right)$ cannot be directly determined applying this empirical model. Furthermore, because all systems analyzed using this model to date involve aromatic donors, it is not known if this relationship is generally true or true only for the type of systems studied. Additionally, these results are based on only a partial probability distribution function fitted to a functional form, not the complete experimentally obtained distribution function.

In addition to the average energy transfer magnitude for strong and weak collisions, the fraction of strong collisions is obtained by fitting the $P\left(E, E^{\prime}\right)$ data to Eqs. (5a) and (5b). Both factors, the magnitude of the energy transferred and the fraction of strong and weak collisions, play a role in determining the overall $P\left(E, E^{\prime}\right)$. The comparison of the energy transfer studies of pyrazine, perfluorobenzene, and methylpyrazine initially seemed to suggest that the magnitude of the strong collisions $(\gamma)$ was related to the proximity of the donor to dissociation threshold while the fraction of strong collisions $(f)$ was related to character of the donor molecules vibrational modes. ${ }^{9}$ However, the above analysis seems to indicate that the mode character is more closely related to the magnitude of the strong and weak collisions, although density of states, to some degree, account for the proximity of the donor to threshold.

Although this study sheds light on several factors that may influence the shape and magnitude of $P\left(E, E^{\prime}\right)$, because so many factors contribute, further experiments and calculations are needed to understand how molecular properties affect $P\left(E, E^{\prime}\right)$.

\section{CONCLUSIONS}

The collisional relaxation of highly vibrationally excited pyrimidine $\left(E_{\mathrm{vib}}=40362 \mathrm{~cm}^{-1}\right)$ by a bath of carbon dioxide has been studied using infrared diode lasers to probe the scattered bath molecules. The nascent rotational populations and recoil velocity distributions for rotational states in the high $J$ tail $(J=58-80)$ of the $00^{\circ} 0$ level of $\mathrm{CO}_{2}$ were measured. The recoil velocity distributions indicate that bath molecules scattered into high $J$ states of the ground vibrational level undergo significant translational and rotational excitations. The quantum state resolved energy transfer probability data have been sorted according to the magnitude of the energy transfer, $\Delta E$, providing the high energy tail of the energy transfer distribution function, $P\left(E, E^{\prime}\right) . P\left(E, E^{\prime}\right)$ for pyrimidine has been compared to $P\left(E, E^{\prime}\right)$ for $\mathrm{C}_{6} \mathrm{~F}_{6}$, pyrazine, and methylpyrazine obtained previously. While collisions that transfer large amounts of energy are evident for all four molecules, the "supercollision" tail behaves differently for each. Double exponential fits to $P\left(E, E^{\prime}\right)$ give the following results. The "fraction" of strong collisions is found to be $f=0.15,0.05,0.05$, and 0.03 , the characteristic energy of strong collisions is found to be $\gamma=1411,1527,2532$, and $3342 \mathrm{~cm}^{-1}$, and the characteristic energy of weak collisions is found to be $\alpha=620,441,296$, and $292 \mathrm{~cm}^{-1}$ for $\mathrm{C}_{6} \mathrm{~F}_{6}$, methylpyrazine, pyrazine, and pyrimidine, respectively.

Trends in these $P\left(E, E^{\prime}\right)$ parameters have been compared to various molecular properties, including number of vibrational modes, number of modes with energy less than $500 \mathrm{~cm}^{-1}$, donor density of states, and proximity to threshold. Comparisons show that the fraction of strong collisions, the average energy transferred in down collisions, and the weak-collision energy increase as the number of vibrational modes, the number of modes with $\nu<500$, and the density of states increases, while the strong-collision frequency is maximized as donor molecules approach the threshold for unimolecular dissociation. Two models have been used to consider the effects of molecular properties on $P\left(E, E^{\prime}\right)$. An $f\left(E, E^{\prime}\right)$ model, which considers both donor mode efficiency and population, most accurately mirrors the shape of $P\left(E, E^{\prime}\right)$ when low-frequency out-of-plane donor modes are assumed to dominate the large $\Delta E$ energy transfer processes, while a model based on Fermi's golden rule indicates that the way in which the donor density of states changes with $\Delta E$ determines the shape of $P\left(E, E^{\prime}\right)$. Clearly many factors influence the shape and magnitude of $P\left(E, E^{\prime}\right)$.

\section{ACKNOWLEDGMENTS}

This work was performed at Brigham Young University with support from the BYU Mentoring Environment Grant. A.M.D., B.J.H., and J.A.J. each acknowledge support from the Department of Chemistry and Biochemistry Undergraduate Research Awards program and the BYU Office of Research and Creative Works Fellowship program.

\footnotetext{
${ }^{1}$ T. Baer and W. L. Hase, Unimolecular Reaction Dynamics: Theory and Experiments (Oxford University Press, New York, 1996).

${ }^{2}$ R. G. Gilbert and S. C. Smith, Theory of Unimolecular and Recombination Reactions (Blackwell Scientific, Oxford, 1990).

${ }^{3}$ G. W. Flynn, C. S. Parmenter, and A. M. Wodtke, J. Phys. Chem. 100,
} 
12817 (1996).

${ }^{4}$ T. Lenzer, K. Luther, K. Reihs, and A. C. Symonds, J. Chem. Phys. 112 4090 (2000); T. Lenzer and K. Luther, Phys. Chem. Chem. Phys. 6, 955 (2004).

${ }^{5}$ U. Hold, T. Lenzer, K. Luther, and A. C. Symonds, J. Chem. Phys. 119, 11192 (2003); U. Grigoleit, T. Lenzer, K. Luther, M. Mutzel, and A. Takahara, Phys. Chem. Chem. Phys. 3, 2191 (2001); H. Frerichs, M. Hollerbach, T. Lenzer, and K. Luther, J. Phys. Chem. A 110, 3179 (2006)

${ }^{6}$ C.-L. Liu, H.-C. Hsu, J.-J. Lyu, and C.-K. Ni, J. Chem. Phys. 123, 131102 (2005); 124, 054302 (2006); H. C. Hsu, C.-L. Liu, J.-J. Lyu, and C.-K. Ni, ibid. 124, 134303 (2006); C.-L. Liu, H.-C. Hsu, J.-J. Lyu, and C.-K. Ni, ibid. 125, 204309 (2006); V. Bernshtein, I. Oref, C.-L. Liu, H.-C. Hsu, and C.-K. Ni, Chem. Phys. Lett. 429, 317 (2006).

${ }^{7}$ C. A. Michaels and G. W. Flynn, J. Chem. Phys. 106, 3558 (1997).

${ }^{8}$ C. A. Michaels, Z. Lin, A. S. Mullin, H. C. Tapalian, and G. W. Flynn, J. Chem. Phys. 106, 7055 (1996)

${ }^{9}$ E. T. Sevy, S. M. Rubin, Z. Lin, and G. W. Flynn, J. Chem. Phys. 113, 4912 (2000)

${ }^{10}$ A. S. Mullin, C. A. Michaels, and G. W. Flynn, J. Chem. Phys. 102, 6032 (1995)

${ }^{11}$ G. W. Flynn and R. E. Weston, Jr., J. Phys. Chem. 97, 8116 (1993).

${ }^{12}$ A. S. Mullin, J. Park, J. Z. Chou, G. W. Flynn, and R. E. Weston, Jr., Chem. Phys. 175, 53 (1993).

${ }^{13}$ E. M. Miller, L. Murat, N. Bennette, M. Hayes, and A. S. Mullin, J. Phys. Chem. A 110, 3266 (2006).

${ }^{14}$ J. Park, Z. Li, A. S. Lemoff, C. Rossi, M. S. Elioff, and A. S. Mullin, J. Phys. Chem. A 106, 3642 (2002).

${ }^{15}$ J. Park, L. Shum, A. S. Lemoff, K. Werner, and A. S. Mullin, J. Chem. Phys. 117, 5221 (2002)

${ }^{16}$ M. S. Elioff, M. Fang, and A. S. Mullin, J. Chem. Phys. 115, 6990 (2001).

${ }^{17}$ G. Lendvay and G. C. Schatz, J. Phys. Chem. 95, 8748 (1991).

${ }^{18}$ G. Lendvay, G. C. Schatz, and L. B. Harding, Faraday Discuss. 102, 389 (1995).

${ }^{19}$ C. Higgins, Q. Ju, N. Seiser, G. W. Flynn, and S. Chapman, J. Phys. Chem. A 105, 2858 (2001); C. Higgins and S. Chapman, ibid. 108, 8009 (2004); V. Bernshtein and I. Oref, J. Chem. Phys. 104, 1958 (1996); D. L. Clarke, K. C. Thompson, and R. G. Gilbert, Chem. Phys. Lett. 182 , 357 (1991); T. Lenzer, K. Luther, J. Troe, R. G. Gilbert, and K. F. Lim, J. Chem. Phys. 103, 626 (1995).

${ }^{20}$ G. Lendvay, J. Phys. Chem. A 101, 9217 (1997).

${ }^{21}$ D. C. Clary, R. G. Gilbert, V. Bernshtein, and I. Oref, Faraday Discuss. 102, 423 (1995).

${ }^{22}$ Z. Li, R. Sansom, S. Bonella, D. F. Coker, and A. S. Mullin, J. Phys. Chem. A 109, 7657 (2005)

${ }^{23}$ T. G. Dietz, M. A. Duncan, A. C. Pulu, and R. E. Smalley, J. Phys. Chem. 86, 4026 (1982)

${ }^{24}$ See EPAPS Document No. E-JCPSA6-128-003803 for a detailed description of the experimental setup. This document can be reached through a direct link in the online article's HTML reference section or via the EPAPS homepage (http://www.aip.org/pubservs/epaps.html).

${ }^{25}$ F. A. Khan, T. G. Kreutz, G. W. Flynn, and R. E. Weston, Jr., J. Chem. Phys. 92, 4876 (1990).

${ }^{26}$ M.-F. Lin, Y. A. Dyakov, C.-M. Tseng, A. M. Mebel, S. H. Lin, Y. T. Lee, and C.-K. Ni, J. Chem. Phys. 124, 84303 (2006).

${ }^{27}$ C. A. Michaels, H. C. Tapalian, Z. Lin, E. T. Sevy, and G. W. Flynn,
Faraday Discuss. 102, 405 (1995).

${ }^{28}$ E. T. Sevy, C. A. Michaels, H. C. Tapalian, and G. W. Flynn, J. Chem. Phys. 112, 5844 (2000); E. T. Sevy, M. A. Muyskens, S. M. Rubin, G. W. Flynn, and J. T. Muckerman, ibid. 112, 5829 (2000).

${ }^{29}$ G. A. Parker and R. T Pack, J. Chem. Phys. 68, 1585 (1978); R. L. Abrams and P. K. Cheo, Appl. Phys. Lett. 15, 177 (1969); P. M. Agrawal and L. M. Raff, J. Chem. Phys. 75, 2163 (1981); R. A. Brownsword, J. S. Salh, and I. W. M. Smith, J. Chem. Soc., Faraday Trans. 91, 191 (1995); R. T Pack, J. Chem. Phys. 70, 3424 (1979); C. Roche, G. Millot, R. Chaux, and R. Saint-Loup, ibid. 101, 2863 (1994).

${ }^{30}$ J. F. Hershberger, J. Z. Chou, G. W. Flynn, and R. E. Weston, Jr., Chem. Phys. Lett. 149, 51 (1988).

${ }^{31}$ C. A. Michaels, A. S. Mullin, and G. W. Flynn, J. Chem. Phys. 102, 6682 (1995); A. J. Sedlacek, R. E. Weston, Jr., and G. W. Flynn, ibid. 94, 6483 (1991).

${ }^{32}$ L. A. Pugh and K. N. Rao, in Molecular Spectroscopy: Modern Research Volume II, edited by K. N. Rao (Academic, New York, 1976), p. 165.

${ }^{33}$ L. S. Rothman, R. R. Gamache, A. Barbe, A. Goldman, J. R. Gillis, L. R. Brown, R. A. Toth, J. M. Flaud, and C. Camy-Peyret, Appl. Opt. 22, 2247 (1983).

${ }^{34}$ D. G. Mitchell, A. M. Johnson, J. A. Johnson, K. A. Judd, K. Kim, M. Mayhew, A. J. Powell, and E. T. Sevy, "Collisional relaxation of the three vibrationally excited difluorobenzene isomers by collisions with $\mathrm{CO}_{2}$ : Effect of donor vibrational mode," J. Phys. Chem. A [Online early access]. DOI: 10.1021/jp0771365.

${ }^{35}$ E. T. Sevy, Ph.D. dissertation, Columbia University, 1999.

${ }^{36}$ D. K. Havey, Q. Liu, Z. Li, M. Elioff, M. Fang, J. Neudel, and A. S. Mullin, J. Phys. Chem. A 111, 2458 (2007).

${ }^{37}$ M. C. Wall, A. S. Lemoff, and A. S. Mullin, J. Phys. Chem. A 102, 9101 (1998); J. Chem. Phys. 111, 7373 (1999); M. C. Wall and A. S. Mullin, ibid. 108, 9658 (1998); M. S. Elioff, M. C. Wall, A. S. Lemoff, and A. S. Mullin, ibid. 110, 5578 (1999).

${ }^{38}$ J. Troe, J. Chem. Phys. 97, 288 (1992).

${ }^{39}$ J. Troe, J. Chem. Phys. 66, 4758 (1977).

${ }^{40}$ J. Troe, J. Chem. Phys. 66, 4745 (1977)

${ }^{41}$ J. Troe, J. Phys. Chem. 83, 114 (1979).

${ }^{42}$ J. F. Durana and J. D. McDonald, J. Chem. Phys. 64, 2518 (1976).

${ }^{43}$ G. Lendvay and G. C. Schatz, J. Phys. Chem. 98, 6530 (1994).

${ }^{44}$ B. J. Hom and E. T. Sevy, (unpublished).

${ }^{45}$ A. M. Duffin, J. A. Johnson, M. A. Muyskens, and E. T. Sevy, J. Phys. Chem. A 111, 13330 (2007).

${ }^{46}$ J. T. Yardley, Introduction to Molecular Energy Transfer (Academic, New York, 1980).

${ }^{47}$ I. W. M. Smith, Kinetics and Dynamics of Elementary Gas Reactions (Butterworths, London, 1980).

${ }^{48}$ J. O. Hirschfelder, C. F. Curtiss, and R. B. Bird, Molecular Theory of Gases and Liquids (Wiley, New York, 1954).

${ }^{49}$ G. Z. Whitten and B. S. Rabinovitch, J. Chem. Phys. 38, 2466 (1963).

${ }^{50}$ E. T. Sevy, M. A. Muyskens, Z. Lin, and G. W. Flynn, J. Phys. Chem. A 104, 10538 (2000)

${ }^{51}$ V. J. Eaton and D. Steele, J. Mol. Spectrosc. 48, 446 (1973); R. A. R. Pearce, D. Steele, and K. Radcliffe, J. Mol. Struct. 15, 409 (1973).

${ }^{52}$ T. Watanabe, H. Shimada, and R. Shimada, Bull. Chem. Soc. Jpn. 55, 2564 (1982)

${ }^{53}$ J. D. Simmons and K. K. Innes, J. Mol. Spectrosc. 14, 190 (1964).

${ }^{54}$ F. Billes, H. Mikosch, and S. Holly, J. Mol. Struct.: THEOCHEM 423, 225 (1998). 